\title{
Thermocapillary Motion of a Newtonian Drop in a Dilute Viscoelastic Fluid
}

\author{
Paolo Capobianchi ${ }^{1}$, Fernando T. Pinho ${ }^{2}$, Marcello Lappa ${ }^{1}$ and Mónica S. N. Oliveira ${ }^{1}$ \\ ${ }^{1}$ James Weir Fluids Laboratory, Department of Mechanical and Aerospace Engineering, University of \\ Strathclyde, Glasgow G1 1XJ, UK
}

${ }^{2}$ CEFT, Departamento de Engenharia Mecânica, Faculdade de Engenharia da Universidade do Porto, Rua Dr. Roberto Frias, 4200-465 Porto, Portugal

\begin{abstract}
In this work we investigate the role played by viscoelasticity on the thermocapillary motion of a deformable Newtonian droplet embedded in an immiscible, otherwise quiescent nonNewtonian fluid. We consider a regime in which inertia and convective transport of energy are both negligible (represented by the limit condition of vanishingly small Reynolds and Marangoni numbers) and free from gravitational effects. A constant temperature gradient is maintained by keeping two opposite sides of the computational domain at different temperatures. Consequently the droplet experiences a motion driven by the mismatch of interfacial stresses induced by the non-uniform temperature distribution on its boundary. The departures from the Newtonian behaviour are quantified via the "thermal" Deborah number, $D e_{T}$, and are accounted for by adopting either the Oldroyd-B model, for relatively small $D e_{T}$, or the FENE-CR constitutive law for a larger range of $D e_{T}$. In addition, the effects of model parameters, such as the concentration parameter $c=1-\beta$ (where $\beta$ is the viscoelastic viscosity ratio), or the extensibility parameter, $L^{2}$ have been studied numerically using a hybrid volume of fluid-level set method. The numerical results show that the steady-state droplet velocity behaves as a monotonically decreasing function of $D e_{T}$, whilst its shape deforms prolately. For increasing values of $D e_{T}$, the viscoelastic stresses show the tendency to be concentrated near the rear stagnation point, contributing to an increase in its local interface curvature.
\end{abstract}

Keywords: Thermocapillary migration, droplet dynamics, viscoelastic effects, LS-VOF

\section{Introduction}

In this work we study the role played by viscoelasticity on the thermocapillary induced motion of a deformable droplet in a polymeric fluid, which arises when the system is subjected to a temperature gradient. There are many industrial and technical applications in which non-uniform heating is applied to a polymeric liquid. Typical examples include (but are not limited to) processes for plastics joining, ${ }^{1,2}$ the heat treatment of polymers aimed at mechanical and tribological properties improvement $\left(\mathrm{Aly}^{3}\right.$ and references therein), the 
welding of plastics, ${ }^{4}$ and thermocapillary actuation of synthetic and biopolymeric fluids for dispersing, mixing and pumping at the microscale,,$^{5}$ amongst many other manufacturing processes in engineering. ${ }^{6,7}$ What sets these examples apart from similar processes using Newtonian fluids is the presence of additional (elastic) stresses in the fluid phase. Indeed, polymeric materials are known for their ability to display both viscous and elastic stresses when subjected to deformation, that is, they exhibit viscoelastic behaviour. Superimposed onto this conceptual characteristic, we often find the presence of immiscible phases, which allow surface-tension driven effects to influence the fluid dynamics.

There have been several works dedicated to the effect of surface tension on polymer liquid dynamics (the interested reader may consider, e.g., Dee and $\mathrm{Sauer}^{8}$ for an exhaustive review). Firstly, whenever two immiscible fluids are in contact, the interfacial tension acts to minimise the surface energy of the system by reducing the area separating the two phases. If the conditions are favourable, the formation of disconnected droplets is a direct consequence of such process of energy minimisation. Then, since frequently the components are also characterised by different densities, gravity can induce subsequent droplet displacement. There is indeed a large body of literature dedicated to the study of the motion of bubbles and drops undergoing sedimentation or flotation in the presence of non-Newtonian fluids under isothermal conditions. To the best of our knowledge, the first documented experiments on the motion of bubbles in viscoelastic fluids is due to Philippoff ${ }^{9}$ who investigated the motion of air bubbles rising through elastic solutions made of rubber dissolved in organic solvents. The experiments revealed that the bubbles assumed a characteristic tear-like shape with the presence of a trailing cusp which was observed to become more pronounced when the fluid relaxation time was increased. For such reason, the behaviour was ascribed to the presence of memory effects. Subsequently, the motion of bubbles rising on otherwise quiescent viscoelastic fluids have been investigated by a number of other authors (see, e.g. ${ }^{10-16}$ ). In particular, Hassager ${ }^{16}$ was the first to realise that the cusp might not be axisymmetric even though the flow conditions were such that there was no apparent motivation to predict such asymmetry. Later Liu et al. ${ }^{17}$ conducted systematic experiments considering air bubbles rising through viscoelastic solutions in containers with different cross-sections (i.e., rounded, squared and rectangular) and discovered that the trailing cusp might actually assume a variety of different shapes. Another interesting phenomenon that can be observed with regard to the motion of both solid and fluid particles translating in a viscoelastic liquid, is the presence of a "negative wake"16. The term 'negative' originates from the fact that although very close to the rear stagnation point the velocity is in the direction of the particle motion, immediately further away from the trailing end the flow reverts direction. On the contrary, when the continuous phase is Newtonian, the velocity in the wake is everywhere in the same direction of the motion of the particle. 
Although buoyancy-induced motion is the most immediate effect one can imagine, other mechanisms based on a different type of forces exerted directly at the droplet interface can be responsible for the activation of the droplet displacement, one typical example being the socalled thermal Marangoni migration. In this case, a non-uniform temperature distribution within the fluid generates interfacial tension gradients. The thermally induced capillary stresses generate flow inside and around the droplet in addition to droplet displacement, which is produced to counter-balance, through viscous (if the fluid is Newtonian) or both viscous and elastic stresses (if the fluid is viscoelastic), the mismatch of the capillary stresses at the interface separating the two immiscible components. In the present work we specifically concentrate on the Marangoni migration of a droplet in the presence of viscoelastic effects (no buoyancy present).

The existing literature on thermal Marangoni droplet migration for the case of Newtonian fluids is vast. Starting from the seminal work by Young et al. ${ }^{18}$ a wealth of research has been published encompassing experimental works both in labs (see for instance ${ }^{19-21}$ ) as well as under microgravity conditions (see, e.g., Hadland et al. ${ }^{22}$ ), analytical solutions ${ }^{23-25}$, numerical simulations based on a variety of numerical methods, such as volume of fluid, ${ }^{26-28}$ levelset, ${ }^{29,30}$ lattice Boltzmann, ${ }^{31,32}$ and hybrid techniques. ${ }^{33,34}$ All these efforts pertain to Newtonian fluids, i.e., there is a clear lack of work on the Marangoni migration of droplets in viscoelastic fluids, which we address in the present work.

Although there is a relevant amount of literature dedicated to the study of thermocapillary flows of fluid layers in the presence of viscoelastic fluids (see, e.g. ${ }^{35-39}$ ), the non-Newtonian thermocapillary problem for bubbles and drops seems to be relatively unexplored. To the best of our knowledge, the analytical solution by Jiménez-Fernández and Crespo ${ }^{40}$ is the only existing attempt where the migration phenomenon induced by Marangoni effects was considered in combination with viscoelasticity under very restrictive conditions. They used a perturbation procedure to find an analytical solution for the case of a non-deformable gas bubble surrounded by an Oldroyd-B fluid in the limit of weak viscoelastic effects (i.e. Deborah numbers smaller than unity), and found that the migration velocity decreases monotonically with the square of the Deborah number for this range of Deborah numbers.

In this work we go well beyond the analysis of Jiménez-Fernández and $\mathrm{Crespo}^{40}$ by taking into account droplet deformability and investigating the role played by the presence of viscoelasticity on the thermocapillary motion of drops over a much wider range of Deborah numbers $\left(D e_{T} \leq 30\right)$. We rely on numerical computations based on a hybrid volume of fluidlevel set method implemented into OpenFOAM for the so-called Stokes regime, i.e. for vanishingly small Reynolds ( $R e$ ) and Marangoni ( $M a)$ numbers, considering a Newtonian 
drop embedded in a viscoelastic continuous fluid modelled using two different constant viscosity constitutive equations (i.e., Oldroyd-B and FENE-CR models).

After this introduction, in Section 2 we describe the problem under examination and list the full set of governing equations. Section 3 focuses on the numerical methodology, followed by the validation of the viscoelastic multiphase solver. The results of the present investigation are then discussed in the subsequent Section 4, in which we first assess the dynamics of droplet migration in an infinitely diluted solution and then describe the effects of polymer concentration for varying Deborah numbers. The impact of other relevant parameters, such as molecule extensibility, are also analysed. Chapter 5 summarises the main findings and conclusions.

\section{Mathematical Formulation}

\subsection{Statement of the problem}

We consider the thermocapillary motion of a Newtonian droplet of radius $R=0.5 \mathrm{~cm}$ surrounded by an otherwise quiescent immiscible viscoelastic liquid contained in a parallelepipedic domain having dimensions $(4.5 \times 4.5 \times 6) \mathrm{cm}^{3}$ with walls on all sides (see Fig. 1). These geometrical constrains are similar to those adopted in the experiments of Hadland et al. ${ }^{22}$ and have also been used in our previous investigations. ${ }^{41,42}$

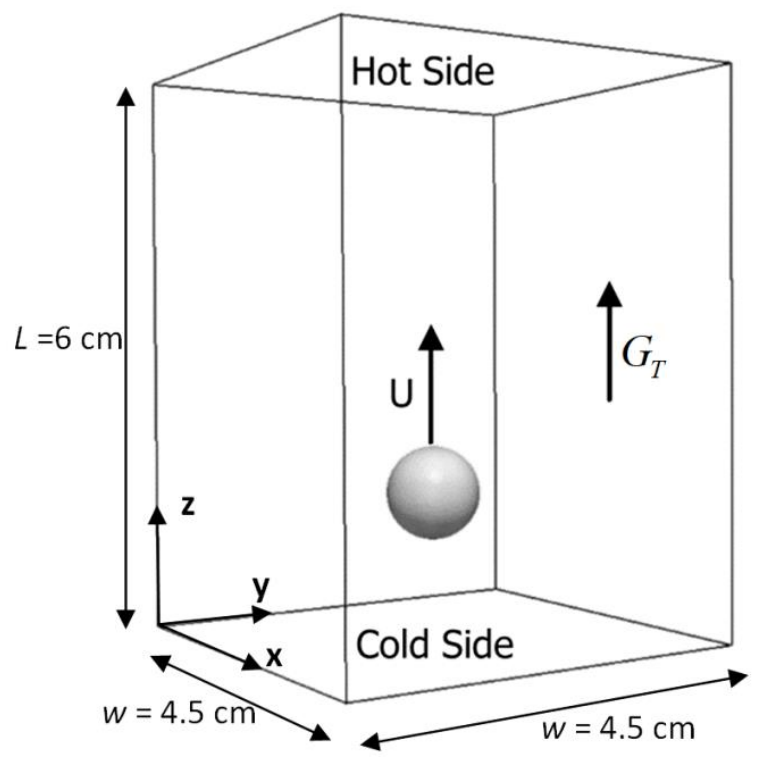

Figure 1: Schematics of the parallelepipedic configuration (equivalent to the experiment of Hadland et al. ${ }^{22}$ ) and coordinate axes considered in the numerical study. 
The outer (matrix) viscoelastic phase is characterised by a shear independent viscosity, $\eta_{0, m}=\eta_{s, m}+\eta_{p, m}$, resulting from the summation of the Newtonian (solvent) contribution, $\eta_{s, m}$, with the viscoelastic (polymer) contribution, $\eta_{p, m}$. A constant temperature gradient, $G_{T}=\left(T_{\text {hot }}-T_{\text {cold }}\right) / L$, is maintained by external means. The fluid properties are assumed to be constant with the exception of the interfacial tension, $\sigma$, which decreases with temperature, $T$, with a constant rate of change, $\sigma_{T}=-d \sigma / d T .^{8}$

In order to introduce the various dimensionless parameters governing the physics of the problem under discussion, we use $R$ as a reference length scale and define the reference velocity scale as $U_{T}=\left|\sigma_{T}\right| R G_{T} / \eta_{0, m}$, having assumed that the thermocapillary stresses at the interface generate velocity gradients of order of magnitude $U_{T} / R$. Pressure and stresses are non-dimensionalised with the characteristic viscous stress, $\eta_{s, m} U_{T} / R$, whilst the temperature is made dimensionless by subtracting the reference value $T_{0}\left(T_{0}\right.$ is defined as the temperature at the centre of mass of the translating drop) and then dividing the temperature difference by the scaling temperature, $R G_{T}$. Finally, time is scaled with the quantity $R / U_{T}$. Using these scalings, we introduce the Reynolds number, $R e=\rho_{m} R U_{T} / \eta_{0, m}$, the Marangoni number, $M a=\rho_{m} c_{p, m} R U_{T} / \kappa_{m}$, and the Prandtl number $\operatorname{Pr}=\eta_{0, m} c_{p, m} / \kappa_{m}$ (these quantities are not independent since $M a=\operatorname{RePr}$ ), where $c_{p, m}$ and $\kappa_{m}$ are heat capacity and thermal conductivity of the outer phase, and the Deborah number defined as $D e_{T}=\lambda U_{T} / R$ (here called "thermal" Deborah number to distinguish from the usual Deborah number definition $D e=\lambda U / R$ adopting the droplet velocity, $U$, instead of $U_{T}$ ). The dimensionless description of the problem is complete with the introduction of the capillary number, $C a=\eta_{0, m} U_{T} / \sigma$ and all the material property ratios between the two phases.

In all the numerical simulations proposed in the present study, we adopted the following values for the dimensionless parameters: $R e=1 \times 10^{-4}, M a=1 \times 10^{-5}$ and $C a=2 \times 10^{-1}$, while the Deborah number, $D e_{T}$ has been varied within the range 0 to 30 . According to the fluid dynamic regime considered, the flow is purely "diffusive" both in terms of momentum and energy, and the temperature field can be assumed to be linear everywhere as the thermal properties of the two fluids are the same (as explained later in Sect. 2.2).

\subsection{Governing Equations}

It is customary to describe the motion of a non-isothermal system composed of two incompressible immiscible fluids by a single set of governing equations. ${ }^{43}$ With such a description, the conservation of mass and momentum, in the absence of gravity and other body forces, can be cast in compact form as: 
$\nabla \cdot \mathbf{u}=0$

$\rho \frac{D \mathbf{u}}{D t}=-\nabla p+\nabla \cdot \Sigma+\mathbf{f}_{\sigma}$

where $\rho$ is the density of the fluid, $\mathbf{u}$ and $t$ represent velocity vector and time, respectively, $p$ is the pressure and $\Sigma=2 \eta_{\mathrm{s}} \mathbf{D}+\tau$ is the total extra stress tensor, comprising the Newtonian contribution, $2 \eta_{\mathrm{s}} \mathbf{D}$, and the viscoelastic extra-stress tensor, $\tau$. In the Newtonian part, $\mathbf{D}=\frac{1}{2}\left(\nabla \mathbf{u}+\nabla \mathbf{u}^{\mathrm{T}}\right)$ is half the rate-of-strain tensor. The symbol $D(\cdot) / D t=\partial(\cdot) / \partial t+\mathbf{u} \cdot \nabla(\cdot)$ denotes the substantial derivative. The last term on the right hand side of Eq. (2), $\mathbf{f}_{\sigma}$, accounts for the capillary, $\left(\mathbf{f}_{\sigma, \mathbf{n}}\right)$, and thermocapillary, $\left(\mathbf{f}_{\sigma, \mathbf{t}}\right)$ forces at the interface:

$\mathbf{f}_{\sigma}=\mathbf{f}_{\sigma, \mathbf{n}}+\mathbf{f}_{\sigma, \mathbf{t}}=\sigma k \mathbf{n} \delta_{\mathrm{S}}+\nabla_{S} \sigma(T) \delta_{S}$

The operator, $\nabla_{S}=\nabla-\mathbf{n}(\mathbf{n} \cdot \nabla)$, is the projection of the operator $\nabla$ to the direction tangent to the interface, $\mathbf{n}$ and $k$ are the normal unit vector and the curvature at the interface, respectively. Finally, the term $\delta_{S}$ is a scalar-valued distribution which identifies the interface. As illustrated by Dee and Sauer, ${ }^{8}$ the surface tension for many polymer molecules can be assumed to vary linearly with temperature:

$\sigma(T)=\sigma\left(T_{0}\right)-\sigma_{T}\left(T-T_{0}\right)$

The mathematical formulation of the problem is completed by including the energy balance equation:

$\rho c_{p} \frac{D T}{D t}=\nabla \cdot(\kappa \nabla T)$,

where $c_{p}$ is the specific heat and $\kappa$ the thermal conductivity coefficient, and the constitutive equation for the viscoelastic stresses.

It is instructive to point out that in the regime of interest (small Marangoni and Reynolds number), the temperature field can be assumed to be linearly uniform everywhere, provided the thermal properties of the two fluids are the same (which is indeed the case in the present work). The problem could therefore have been addressed leaving aside the energy equation. Nonetheless, since the solution of the energy equation did not produce specific problems for the present computations (not contributing to increase significantly the computational cost), the equation has been retained in the solving algorithm. 
To model the viscoelastic fluid behaviour, we consider both the Oldroyd- ${ }^{44}$ and the FENE-CR ${ }^{45}$ constitutive models (see e.g. ${ }^{7,46-49}$ ) generally represented by the following evolution equation for the conformation tensor:

$\lambda \stackrel{\nabla}{\mathbf{A}}=-f(\operatorname{tr}(\mathbf{A}))(\mathbf{A}-\mathbf{I})$

where $\stackrel{\nabla}{\mathrm{A}}=D \mathrm{~A} / D t-\left(\nabla \mathbf{u}^{\mathrm{T}} \cdot \mathbf{A}+\mathbf{A} \cdot \nabla \mathbf{u}\right)$ represents the upper-convected derivative of the socalled conformation tensor A . For the FENE-CR model, the function $f$ appearing in Eq. (6) reads:

$f(\operatorname{tr}(\mathbf{A}))=\frac{L^{2}}{L^{2}-\operatorname{tr}(\mathbf{A})}$

where $L^{2}$ is the so-called extensibility parameter of the polymer molecule. The specific condition $f(\operatorname{tr}(\mathbf{A}))=1$ corresponds ideally to a polymer molecule with infinite extension, i.e. $L^{2} \rightarrow \infty$, for which the standard Oldroyd-B model is recovered. The elastic extra-stress tensor $\tau$ included in the momentum equation can finally be obtained from the conformation tensor via the relationship: ${ }^{50}$

$\tau=\frac{\eta_{\mathrm{p}}}{\lambda} f(\operatorname{tr}(\mathbf{A}))(\mathbf{A}-\mathbf{I})$

Although the governing equations are solved in dimensional form, it is useful to show their non-dimensional counterpart in order to appreciate the dependence of the considered problem on the non-dimensional parameters introduced in Sect. 2.1. It is worth noting that all the material properties appearing in the governing equations may, in general, vary across the interface. For this reason, a generic fluid property, $\chi$, is generally expressed as a linear combination (although other types of combination are sometimes used, see e.g., Capobianchi et al. ${ }^{51}$ ) of the values assumed inside the two phases using the volume fraction $\alpha_{k}$ as combination parameter ( $\alpha_{k}$ being 0 or 1 depending on the considered fluid):

$\chi=\alpha_{k} \chi_{d}+\left(1-\alpha_{k}\right) \chi_{m}$

Considering the generic material property of the matrix fluid $\chi_{m}$ as the reference for the $\chi$ property, fluid properties can be written in dimensionless form as $\chi_{r}=\alpha_{k} \chi_{d} / \chi_{m}+\left(1-\alpha_{k}\right)$. It is worth pointing out that in the present investigation we assumed that the two fluids have the same properties (except for the viscoelastic properties, for obvious reasons). Therefore, in the present case, Eq. 9 becomes relevant only when applied to the values of $\eta_{p}$ and $\lambda$. 
Taking into account the dimensionless characteristic numbers defined in Sect. 2.1, the following set of dimensionless governing equations is obtained

$$
\begin{aligned}
& \rho_{r} R e \frac{D \mathbf{u}}{D t}=-\nabla p+\nabla \cdot\left[(1-c) \eta_{0, r} \mathbf{D}+\tau\right]+\frac{1}{C a} k \mathbf{n} \delta_{S}+\left(T-T_{0}\right) k \mathbf{n} \delta_{S}+(\mathbf{I}-\mathbf{n n}) \nabla T \delta_{S} \\
& \rho_{r} c_{p, r} \frac{D T}{D t}=\frac{1}{M a} \nabla \cdot\left(\kappa_{r} \nabla T\right) \\
& \nabla=-\frac{1}{\lambda_{r} D e_{T}} f(\operatorname{tr}(\mathbf{A}))(\mathbf{A}-\mathbf{I}) \\
& \tau=\frac{c \eta_{r, 0}}{\lambda_{r} D e_{T}} f(\operatorname{tr}(\mathbf{A}))(\mathbf{A}-\mathbf{I})
\end{aligned}
$$

where $c=\eta_{p, m} / \eta_{0, m}$ is a parameter proportional to the concentration of polymer molecules dispersed into the solution, related to the viscoelastic viscosity ratio, $\beta=\eta_{s, m} / \eta_{0, m}$, by the simple relation $c=1-\beta$.

In the following, not to increase excessively the complexity of the mathematical model, we assume that the capillary number is small enough to guarantee that the condition $\frac{1}{C a} k \mathbf{n} \delta_{S} \gg\left(T-T_{0}\right) k \mathbf{n} \delta_{S}$ is satisfied. Accordingly, the fourth term on the right-hand-side of Eq. 10 is neglected in the ensuing calculations.

It is worth emphasising that, although the contribution of the neglected term discussed above is of the same order of magnitude of the $5^{\text {th }}$ term of the same equation (note that both are proportional to the temperature difference established at the drop interface), their effects on the droplet dynamics are profoundly different. In fact, the $4^{\text {th }}$ term is essentially a force acting along a direction perpendicular to the surface of the droplet. It can be seen as a temperatureinduced 'perturbation' of the local surface tension and it is generally balanced by a corresponding change in the distribution of normal stresses at the interface. Its effects are limited to minor variations in the shape of the droplet and, if its relative strength is negligible compared to the normal stress associated with $\sigma\left(T_{0}\right)$. The $5^{\text {th }}$ term, on the other hand, is a force acting tangentially to the interface. It has to be balanced by the tangential viscous stresses produced in the fluid and plays an important role in propelling the droplet, being the main force driving the dynamics of interest.

\section{Numerical method}

\subsection{The hybrid Level-Set VOF solver}


The numerical results presented in the following sections were obtained using a thermocapillary solver ${ }^{42}$ based on a hybrid level-set volume of fluid implemented in the OpenFOAM code. ${ }^{52}$ The solver is based on the original formulation of Albadawi et al. ${ }^{53}$ and has been thoroughly described and tested in Capobianchi et al., ${ }^{41,42,51}$ hence here we provide only a general overview.

In the standard algebraic volume of fluid (VOF) "interFoam" solver available in OpenFOAM, the volume fraction phase, $\alpha_{k}$, is advected using a surface compression approach $^{54}$ in combination with high-resolution numerical schemes, thereby making unnecessary a geometric reconstruction of the interface. The main advantage of such procedure lies in its robustness and ability to handle complex interfaces with limited computational cost. This approach uses the specific variant of Albadawi et al., ${ }^{53}$ who combined the excellent mass-preserving properties of the VOF with the ability of the levelset method to improve the representation of the interface. In the following, we describe briefly the simplified LS-VOF methodology of Albadawi et al. ${ }^{53}$ as implemented in OpenFoam by Yamamoto et al. ${ }^{52}$

In standard LS-VOF codes, the volume fraction $\alpha_{k}$ and the level-set function $\varphi_{k}$ are integrated in time by means of the following advection equation on the basis of an operator splitting technique (see e.g., Tryggvason et al. ${ }^{55}$ ):

$$
\frac{\partial G_{k}}{\partial t}+\nabla \cdot\left(G_{k} \mathbf{u}\right)=0
$$

where $G_{k}$ is either the volume fraction (i.e., $G_{k}=\alpha_{k}$ ) or the level-set function (i.e., $G_{k}=\varphi_{k}$ ). Afterward, the interface is geometrically reconstructed from the volume fraction field, and the curvature is subsequently updated by means of the level-set function. As referred to above, since in the interFoam code of OpenFOAM there is no geometric reconstruction of the interface, such a strategy cannot be applied in a straightforward manner. In the alternative methodology proposed by Albadawi et al. ${ }^{53}$ used here, the initial level-set function is computed in a simplified way from the volume fraction

$\varphi_{0, k}=\left(2 \alpha_{k}-1\right) \Delta$,

where $\Delta$ is a dimensionless number which depends on the mesh resolution (Albadawi et al. ${ }^{53}$ ), set as $\Delta=0.75 \Delta x$, where $\Delta x$ is the grid resolution. Subsequently, a re-initialisation equation is solved for $\varphi_{k}$ with the initial condition set as $\varphi_{k}(\mathbf{x}, 0)=\varphi_{0, k}(\mathbf{x})$

$\frac{\partial \varphi_{k}}{\partial \tau_{f}}=\operatorname{sgn}\left(\varphi_{0, k}\right)\left(1-\left|\nabla \varphi_{k}\right|\right)$ 
where $\operatorname{sgn}\left(\varphi_{0, k}\right)=\varphi_{0, k} /\left|\varphi_{0, k}\right|$ is the sign function and $\tau_{f}=0.1 \Delta x$ is a fictitious time. Subsequently, the field $\varphi_{k}$ is used to evaluate the interface normal and curvature, which in turn are used for the calculation of the capillary force $\mathbf{f}_{\sigma}$. According to our experience, the hybrid code of Yamamoto et al. ${ }^{52}$ appears to be more accurate than the original interFoam solver, nevertheless we further improved its performance by introducing a smoothing strategy (notice that by definition the level-set function is per se already a smooth function) to better describe the interface. This simple strategy is based on the solution of a purely diffusive equation for the level-set function for a prefixed number of mollification cycles $m$

$\varphi_{k, m o l}^{m+1}=\varphi_{k, m o l}^{m}+\left(\nabla^{2} \varphi_{k, m o l}^{m}\right) \Delta \tau_{m o l}$

where $\tau_{m o l}$ is a fictitious time defined by means of stability considerations (the reader is referred to Capobianchi et al. ${ }^{42}$ for a more detailed description of the method) that depends on the grid spacing. Once the smoothed level-set function, $\varphi_{k, m o l}$, is known, the normal unit vector and curvature at the interface are evaluated in the usual manner

$$
\begin{aligned}
& \mathbf{n}\left(\varphi_{k, \text { mol }}\right)=-\frac{\nabla \varphi_{k, m o l}}{\left|\nabla \varphi_{k, m o l}\right|}, \\
& k\left(\varphi_{k, m o l}\right)=\nabla \cdot \mathbf{n}\left(\varphi_{k, m o l}\right) .
\end{aligned}
$$

Finally, the terms accounting for the capillary and thermocapillary forces (Eq. 3) are computed using Eqs. (13) and (14), yielding the following form of the momentum equation:

$$
\begin{aligned}
\rho \frac{D \mathbf{u}}{D t} & =-\nabla p+\nabla \cdot\left(\eta_{\mathrm{s}} \mathbf{D}+\tau\right)+\sigma k\left(\varphi_{k, m o l}\right) I\left(\varphi_{k, m o l}\right) \nabla \varphi_{k, m o l} \\
& +\sigma_{T}\left(\mathbf{I}-\mathbf{n}\left(\varphi_{k, \text { mol }}\right) \mathbf{n}\left(\varphi_{k, m o l}\right)\right) \nabla T\left|\nabla \alpha_{k}\right|
\end{aligned}
$$

where $\quad I\left(\varphi_{k}\right)= \begin{cases}0 & \text { if }\left|\varphi_{k}\right|>\varepsilon \\ \frac{1}{2 \varepsilon}\left(1+\cos \left(\frac{\varphi_{k} \pi}{\varepsilon}\right)\right) & \text { if }\left|\varphi_{k}\right| \leq \varepsilon\end{cases}$

is an "indicator function" and $\varepsilon$ is an empirical parameter such that $\varepsilon=1.5 \Delta x$, in accordance to previous works (see, e.g., ${ }^{56-58}$ ).

\subsection{Viscoelastic solver}

For the solution of the viscoelastic flow problem, we used a multiphase version of the viscoelasticFluidFoam solver originally developed by Favero et al. (2010). ${ }^{59}$ Our solution 
procedure is formally identical except in the approach used to update the stress tensor (formalised by Eq. 8) before solving the momentum equation, since for our purposes we found advisable to re-formulate the governing equation in terms of conformation tensor. Additional specific information on the treatment of the viscoelastic stress term can be found in Appendix A, with the remainder of this section providing detailed information about the validation strategy used.

Towards this end, we considered the deformation of a two-dimensional droplet subjected to shearing, inertialess motion inside a planar Couette cell either in the presence of one or two viscoelastic phases (see, for instance, the cases discussed in Pillapakkam and Singh $^{60}$ and in Chinyoka et al. ${ }^{61}$ ).

A circular droplet of radius $R$ is placed at the centre of a domain of height $h$ and width $\pi h$ (see Fig. 2) delimited by two parallel walls moving in opposite directions along the $x$-axis direction with a constant velocity of magnitude $U_{0}$. At the moving walls, we imposed no-slip and no-through flow boundary conditions for the velocity, while the wall pressure is assigned the values calculated at the nearest neighbour cells centre (i.e., denoted as the "zeroGradient" boundary condition in OpenFOAM). At the two lateral boundaries, periodic conditions have been applied. The flow field is initialised by imposing fully developed uniform shear flow in the whole domain (including also the interior of the droplet) and zero viscoelastic stresses (i.e., $\mathbf{A}=\mathbf{I}$ ). Even though the initial condition for the stresses is not consistent with the imposed velocity field, this does not impact the steady state solution as long as the Capillary number is low enough to guarantee relatively moderate droplet deformations (for more details about this assumption, see again Chinyoka et al. ${ }^{61}$ ). In all the simulations, we employed a uniformly spaced mesh of resolution $\Delta x=R / 25$. 


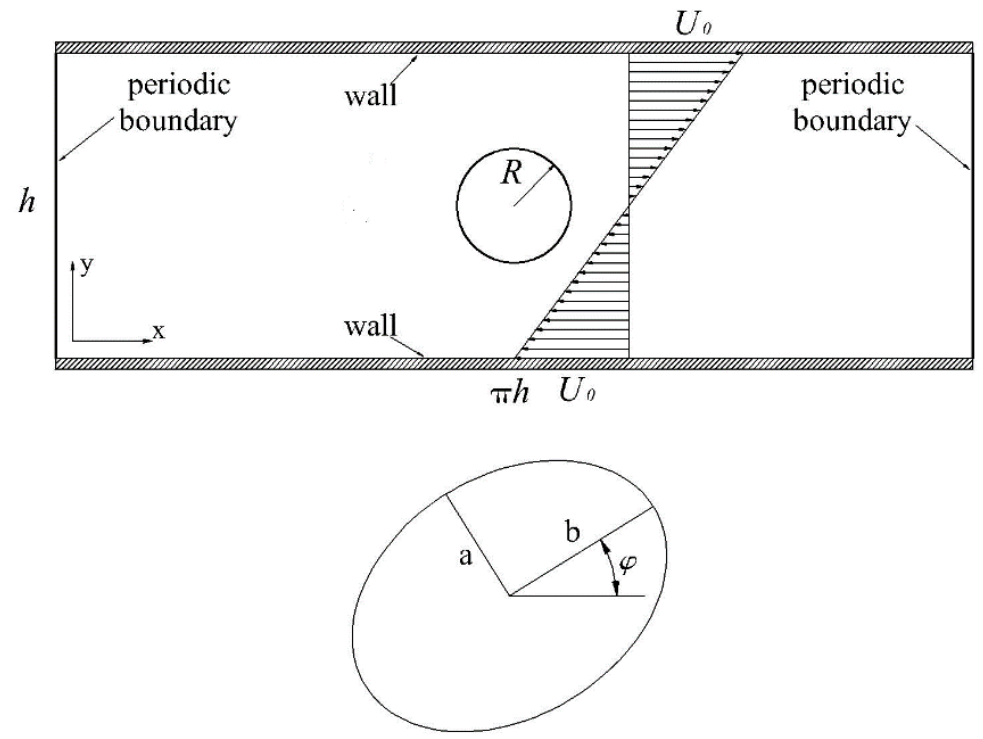

Figure 2: Schematic of the domain and initial flow conditions (top) considered for the shear flow validation case. Steady state deformed droplet shape (bottom), showing the major and minor axes used to calculate the deformation parameter, $D$, and the orientation angle, $\varphi$.

The effect of viscoelasticity on the droplet deformation was taken into account in the framework of the Oldroyd-B viscoelastic model considering the four possible different flow configurations: Newtonian droplet in a Newtonian phase $(\mathrm{N}-\mathrm{N})$, viscoelastic droplet and Newtonian matrix phase (V-N) and the other two possible combinations, N-V and V-V. The flow conditions are such that, $R e=\rho_{m} \dot{\gamma} R^{2} / \eta_{0, m}=3 \times 10^{-4}, \quad C a=\eta_{0, m} \dot{\gamma} R / \sigma=0.24$, $D e_{i}=\lambda_{i} \dot{\gamma}=0.4, \beta=\eta_{s, i} / \eta_{0, i}=0.5$ (the subscript " $i$ " stands for " $m$ " or " $d$ " depending on whether the viscoelastic phase is the matrix or the droplet), where $\dot{\gamma}=2 U_{0} / h$ is the imposed shear rate. The two fluids are assumed to have the same density and viscosity (i.e. $\eta_{0, d} / \eta_{0, m}=1$ and $\left.\rho_{0, d} / \rho_{0, m}=1\right)$ and the same $\beta$ when both phases are viscoelastic, while the geometric confinement is set to $R / h=0.125$ as in Chinyoka et al. ${ }^{61}$ ).

Table I summarises the steady state results of the orientation angle, $\varphi$, and the deformation parameter $D=(a-b) /(a+b)$, with $a$ and $b$ being the major and minor axes as indicated in Fig. 2. The present results are in good agreement with those obtained by Chinyoka et al. ${ }^{61}$ with a maximum relative difference of $\sim 4 \%$ both in terms of deformation and orientation angle. 
Table I: Comparison between our steady state results and those of Chinyoka et al. ${ }^{61}$ in terms of droplet deformation, $D$, and orientation angle, $\varphi$.

\begin{tabular}{ccccccc}
\hline \hline & \multicolumn{2}{c}{ Chinyoka et al. ${ }^{61}$} & \multicolumn{2}{c}{ Current } & \multicolumn{2}{c}{ Deviation $\%$} \\
\hline & $D$ & $\varphi\left[^{\circ}\right]$ & $D$ & $\varphi\left[^{\circ}\right]$ & $D$ & $\varphi\left[^{\circ}\right]$ \\
\hline $\mathrm{N}-\mathrm{N}$ & 0.288 & 32.3 & 0.283 & 31.8 & $<2$ & $<2$ \\
\hline $\mathrm{V}-\mathrm{N}$ & 0.282 & 31.2 & 0.271 & 31.9 & $<4$ & $\approx 2$ \\
\hline $\mathrm{N}-\mathrm{V}$ & 0.265 & 28.2 & 0.265 & 28.2 & $<1$ & $\approx 0$ \\
\hline $\mathrm{V}-\mathrm{V}$ & 0.26 & 28.2 & 0.258 & 29.2 & $<1$ & $<4$ \\
\hline \hline
\end{tabular}

\section{Results}

As explained in the introduction, the objective is to investigate the role of elasticity on the thermocapillary motion of a droplet in the absence of gravity. We performed a series of threedimensional simulations for a single Newtonian drop translating in an otherwise stagnant viscoelastic fluid (c.f. the 3D configuration shown in Fig. 1.) using an adaptive mesh with resolution $\Delta x=R / 28$ in the region of the droplet. The outcomes of the related meshrefinement study performed to guarantee grid-independent 3D solutions are described in Appendix B.

To model the viscoelastic phase and investigate a broad range of Deborah numbers, the simulations were carried out considering a) the Oldroyd-B model, for relatively small Deborah numbers (up to $D e_{T}=3.75$ ), and b) the FENE-CR model, for larger Deborah numbers (up to $D e_{T}=30$ ). This twofold choice is dictated by the presence of an unphysical singularity in the solution of the Oldroyd-B model in extensional flows, which in this specific case develops at the rear stagnation point of the drop (the reader being referred, e.g., to ${ }^{7,62-64}$ for additional insights). In the following sections, we discuss the effect of the various relevant dimensionless numbers (namely $D e_{T}, c$ and $L^{2}$ ) on the droplet dynamics and in particular on the migration and deformation of the droplet.

\subsection{Infinitely dilute solution}

First, we consider the case of the Oldroyd-B fluid $\left(L^{2} \rightarrow \infty\right)$ in the limiting situation in which the concentration of polymer molecules in the solution is infinitely small, i.e., $c \rightarrow 0$ (in 
practice, we set $c=0$ in our simulations, which corresponds to a Newtonian fluid). However, we can still determine the conformation tensor evolution by solving Eq. (12), thus allowing us to separate effects and therefore to better understand the dynamics of droplet motion and deformation, since, in this case, we are able to observe the deformation and orientation of polymer molecules as they flow around the droplet without taking into account the presence of viscoelastic stresses that would modify the flow field and the droplet shape. Then, in Sect. 4.2 the presence of viscoelastic stresses will be analysed corresponding to finite (non-zero) values of $c$.

Figure $3 \mathrm{a}$ shows the temporal evolution of the scaled droplet velocity for $c=0$ and $D e_{T}=3.75$. After a relatively short transient, the droplet velocity approaches the theoretical value obtained by Young et al. ${ }^{18}$ for Newtonian fluids under the assumption of negligible inertia and negligible convective transport of energy, given by

$$
U_{Y G B}=\frac{2\left|\sigma_{T}\right| G_{T} R / \eta_{0, m}}{\left(2+\frac{\kappa_{d}}{\kappa_{m}}\right)\left(2+3 \frac{\eta_{d}}{\eta_{0, m}}\right)}
$$

In this case the shape is nearly spherical (for a discussion on the small departures from the exact shape please refer to appendix B). In particular, to analyse the distribution of the conformation tensor at the droplet interface, we consider the centreplane $x / w=0.5$ passing through the centre of the drop, as shown in Fig. $3 \mathrm{~b}$.

The three components of the conformation tensor on such region are reported in Fig. 4 (qualitatively similar results were obtained for other planes passing through the axis of the drop). We do not display the $x x$-component as we found it to remain nearly constant throughout the reference interface. 


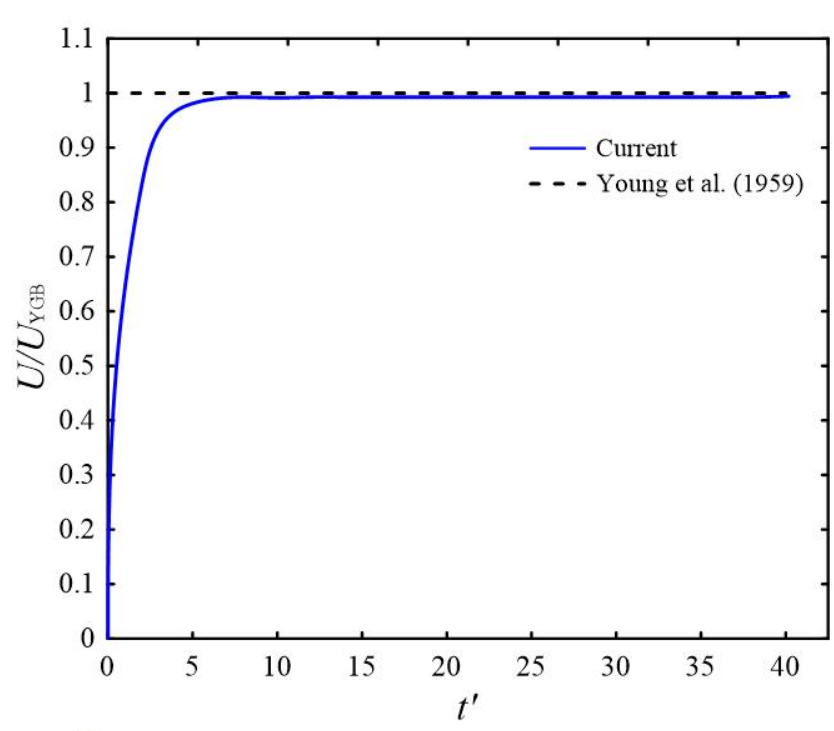

a)

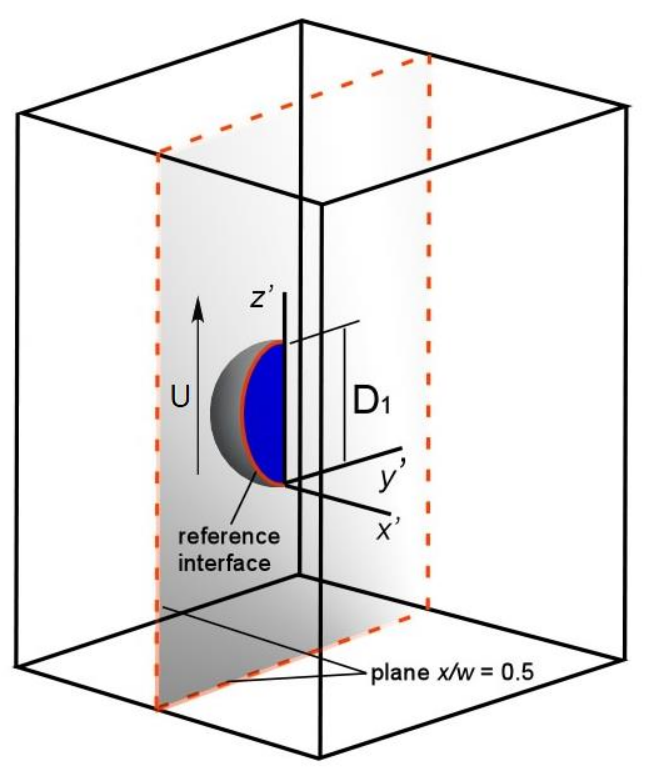

b)

Figure 3: (a) Scaled droplet migration velocity as a function of the dimensionless time for the Oldroyd-B model with $D e_{T}=3.75$ and $c=0$. The dashed line indicates the theoretical steady state value obtained by Young et al. ${ }^{18}$ for Newtonian fluids. (b) Sketch of the flow domain and the droplet cut by the plane $x / w=0.5$ (for the sake of representation, only the portion of the drop where the reference interface (contour $\alpha_{k}=0.5$ ) is taken is shown). The $\left(x^{\prime}, y^{\prime}, z^{\prime}\right)$ coordinate system we consider is also shown and is not fixed in space but advected with the drop, and has the origin of the axes coincident with the rear stagnation point.

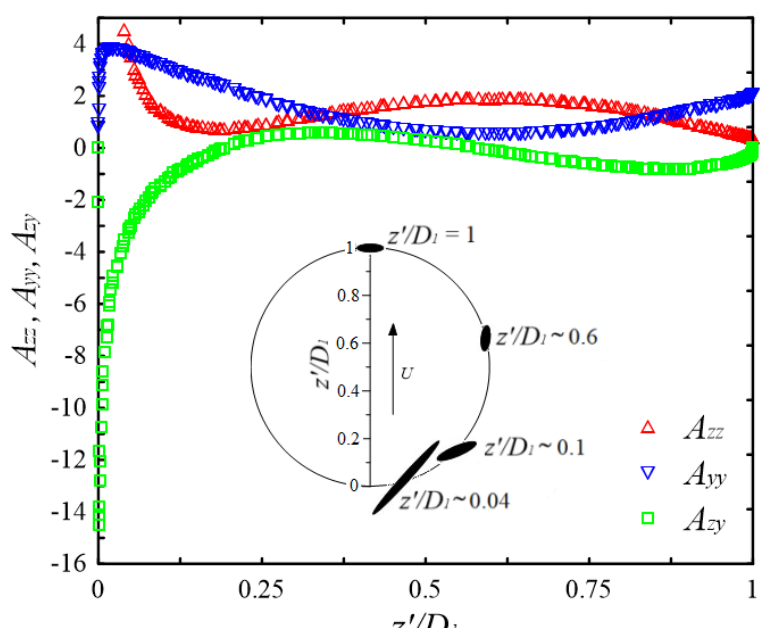

a)

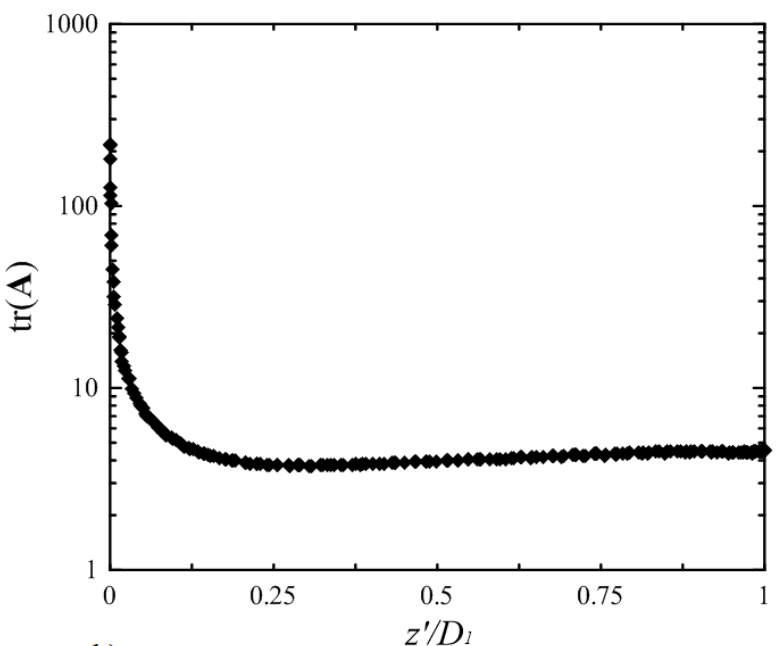

b)

Figure 4: Conformation tensor along the droplet reference interface for the Oldroyd-B model with $D e_{T}=3.75$ and $c=0$, showing the normal and shear components $A_{z z}, A_{y y}, A_{z y}$ (a) and its trace (b). $z^{\prime}$ is taken in such a way that $z^{\prime} / D_{1}=0$ corresponds to the rear stagnation point, 
and $z^{\prime} / D_{1}=1$ to the front stagnation point (as shown in Fig. 3b and in the inset of Fig. 4a). The component $A_{z z}$ for $z^{\prime} / D_{1}<0.04$ has been cut off to make the representation more intelligible, since its maximum value is far larger than the maximum value of the other components. In the inset of plot (a) the conformation tensor has been represented at four different locations of the interface by drawing ellipses that have major and minor axes parallel to the eigenvectors of $\mathbf{A}$ and lengths proportional to the corresponding eigenvalues.

To provide a direct visual representation of the deformation and orientation of the polymer molecule as it flows around the droplet, in Fig. 4a we have also represented the conformation tensor by including ellipses with axes parallel to the principal axes defined by the eigenvectors of $\mathbf{A}$ (while the extensions are proportional to the corresponding eigenvalues, see, e.g. Harlen, $2002^{65}$ ). We now analyse the polymer molecule dynamics as it moves from the front $\left(z^{\prime} / D_{1}=1\right)$ towards the rear stagnation point $\left(z^{\prime} / D_{1}=0\right)$. It can be seen that as the polymer chains approach the front stagnation point, they initially experience a bi-axial extension along the $y$-direction while being compressed along the $z$-direction (cf. the ellipsoid shown at $\left.z^{\prime} / D_{1}=1\right)$. Subsequently, when the molecules move further towards the rear of the drop $A_{y y}$ gradually decreases to a minimum (for $z^{\prime} / D_{1} \sim 0.6$ ) where the deformation is "compressive" $\left(A_{y y}<1\right)$. On the other hand, $A_{z z}$ follows the opposite trend: it gradually increases, becomes extensional and reaches a peak approximately at the same location where the other component attains its minimum value (i.e. at $z^{\prime} / D_{1} \sim 0.6$ ). It is worth noticing that for $z^{\prime} / D_{1} \sim 0.6, A_{z y}$ is approximately zero. As the molecules move further towards the rear region, they extend along the $y$-direction, with $A_{y y}$ reaching a maximum value and finally vanishing as they approach the rear stagnation point. On the other hand, $A_{z z}$ decreases and reaches a minimum at $z^{\prime} / D_{1} \sim 0.1$ (where $A_{z z}$ is close to unity, indicating a nearly relaxed state along the $z$-direction) after which the deformation suddenly increases and eventually reaches its largest value when $z^{\prime} / D_{1}$ is almost zero. Note that the values for small $z^{\prime} / D_{1}$ are not shown in Fig. 4 for sake of representation (cf. the caption in Fig. 4). Regarding the shear component, $A_{z y}$, it is worth highlighting its sudden decrease near the rear region, which is responsible for the change of the orientation of the molecules along the $z$-direction. As illustrated by the ellipse for $z^{\prime} / D_{1} \sim 0.04$, although the polymer filaments are relatively close to the rear region, their orientation is still far from being aligned with the $z$-axis. The large shear component will guarantee that the molecules are oriented in the direction of $z$ axis when they reach the rear stagnation point.

Figure $4 \mathrm{~b}$ shows the distribution of the trace of the conformation tensor, $\operatorname{tr}(\mathbf{A})$, along the same reference interface providing an indication of the degree of stretching of the molecules. We notice that the largest deformation occurs in a narrow region near the rear stagnation point $\left(z^{\prime} / D_{1} \sim 0\right)$, where the flow field is essentially a uniaxial straining flow. The occurrence of the largest molecular stretching at the rear of the droplet is qualitatively similar 
to what can be observed for the analogous case of the gravitational motion of a Newtonian drop in a viscoelastic liquid in isothermal conditions, where the drop assumes a tear-drop shape with a characteristic pointed tail (the interested reader being referred to the collection of experimental images available in $\mathrm{Chhabra}^{6}$ or the numerical results of Pillapakkam et al. $\left.{ }^{66}\right)$. This suggests that the viscoelastic stresses tend to concentrate in a small area around the rear of the drop, with significant consequences on the morphological evolution of the droplet and distribution of the velocity field near the rear stagnation point.

\subsection{The effect of the polymer concentration}

In this section we focus on the effect of finite, non-vanishingly small, polymer concentrations. In contrast to the case addressed in the previous section, the molecular deformation associated with the flow field generates viscoelastic stresses, which are related to the presence of polymer molecules in the viscoelastic phase.

Fig. 5a shows the comparison between the normal components of the conformation tensor for three different values of the parameter $c, c=0, c=0.5$ and $c=0.89$, for a fixed value of the Deborah number, $D e_{T}=3.75$. Irrespective of the value of $c$, the trends for $A_{z z}$ remain qualitatively similar to those discussed in Sect. 4.1, with the main quantitative difference being a small increment of the peak observed in the region corresponding to the front half of the droplet $\left(0.5<z^{\prime} / D_{1}<1\right)$ as the concentration is increased. On the contrary, $A_{y y}$ remains substantially unvaried in the front half, then, as the polymer molecules move towards the rear region, the trends appear remarkably different. In particular, for $c=0$, the maximum extent of the elongation along the $y$-direction appears very close to the rear stagnation point. As the polymer concentration is increased, the maximum value of $A_{y y}$ is gradually shifted towards higher values of $z^{\prime} / D_{1}$. 


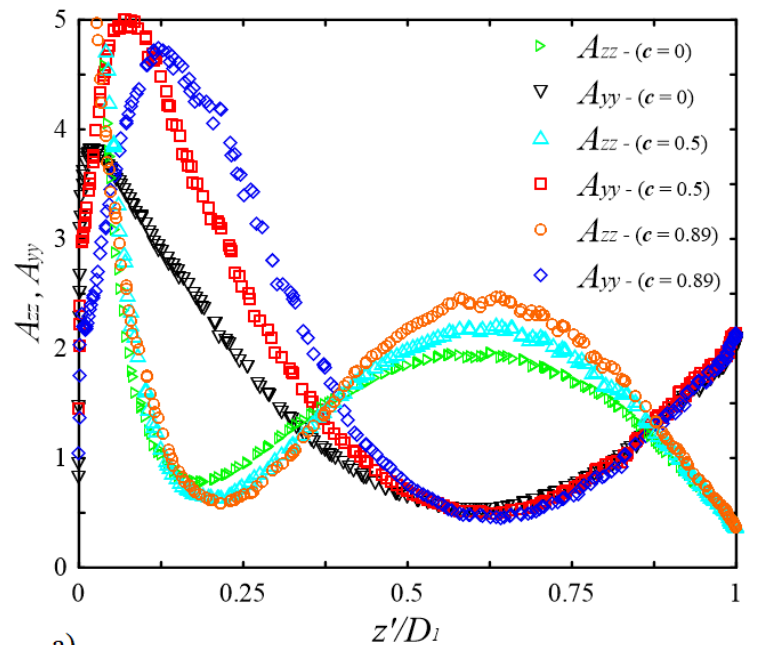

a)

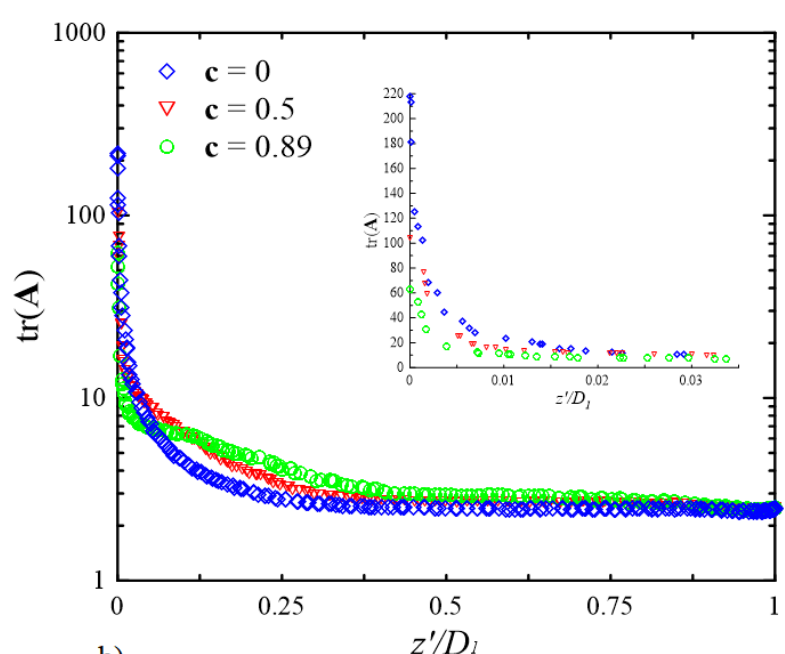

b)

Figure 5: (a) Normal components of the conformation tensor $A_{z z}, A_{y y}$ and (b) trace of the conformation tensor $\mathbf{A}$ along the droplet reference interface obtained using the Oldroyd-B model for three different polymer molecules concentrations $(c=0,0.5$ and 0.89$)$ and for $D e_{T}=3.75$. The inset in figure (b) shows the trace of $\mathbf{A}$ in the region near the rear stagnation point in linear scale.

Figure $5 \mathrm{~b}$ shows the trace of $\mathbf{A}$ for the same three values of $c$. As the molecules approach the rear of the drop, $\operatorname{tr}(\mathbf{A})$ is smaller for higher concentration (at the stagnation point, the value of $\operatorname{tr}(\mathbf{A})$ for $c=0.89$ is about four times smaller than that for the case $c=0$ ). This means that the maximum elongation decreases when the concentration of polymer increases. It is worth noticing that although the results are obtained at a constant thermal Deborah number, the alternative Deborah number evaluated using the actual droplet velocity (typically used in the literature for the case of buoyant-driven isothermal flows) would decrease for increasing values of $c$ since, as it will appear clear soon, the migration velocity is a monotonic decreasing function of the polymer concentration. In addition there are a number of influential factors affecting the flow field near the rear of the droplet, as tentatively illustrated in the following. As in the considered simulations the total viscosity is maintained constant, the reduction of the Newtonian solvent contribution implies a reduced solvent viscosity $\left(\eta_{s}=(1-c) \eta_{0}\right)$, which, in turn, leads to a reduction of the Newtonian contribution to the total stress. Simultaneously, the polymer contribution generates increasingly higher viscoelastic stresses, which are mainly concentrated in a small area near the rear stagnation point where they are essentially extensional. These stresses "pull back" the droplet interface and, if they are large enough to overcome the capillary force, they can contribute to increase the local interface curvature. In turn, resulting in a localised increment of the pressure jump across the droplet interface affecting the flow conditions near the rear region of the droplet.

The influence of polymer concentration on the droplet velocity is illustrated in Fig. 6a, where the scaled droplet speed is shown as a function of the dimensionless time for a constant value 
of $D e_{T}=3.75$. Initially, the droplet speed increases rapidly, exhibiting an overshoot before reaching steady state conditions. We notice that the magnitude of the velocity peak depends on the parameter $c$, becoming larger when $c$ is increased. Note also that for higher values of $c$ the overshoot is followed by undershoot before the velocity tends to the steady state value. Such behaviour can be understood considering that the viscoelastic stresses need a certain amount of time to develop, and, hence (at least in an initial stage) the stresses at the interface are mainly of a "Newtonian nature". In other words, since the concentration is given by the ratio of the polymer viscosity to the total viscosity, having assumed the latter property constant for each simulation, a larger value of $c$ implies a smaller solvent viscosity, thus the Newtonian stresses prevailing at the first stage of the transient determine the observed behaviour. The corresponding steady state velocity for the cases under discussion are shown in Fig. 6b, which shows that when the amount of polymer is increased, the droplet speed decreases monotonically.

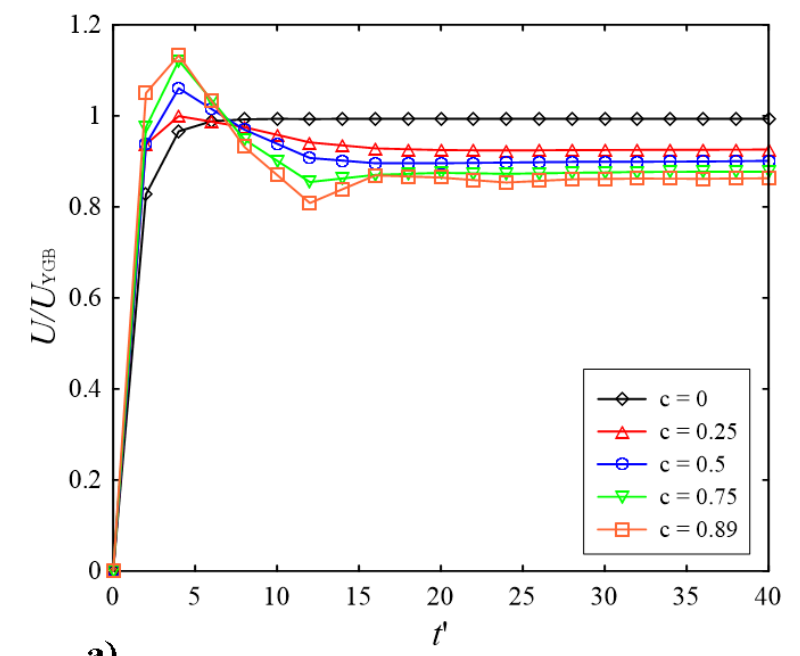

a)

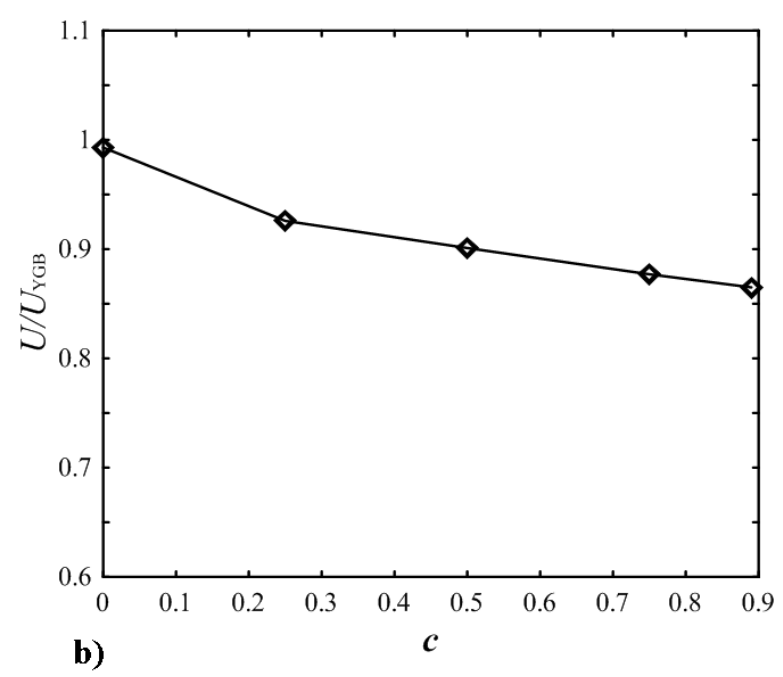

b)

Figure 6: Effect of concentration on the droplet migration velocity. (a) Time evolution of the scaled droplet speed for different polymer concentrations (the points are taken at every $0.5 \mathrm{~s}$ and the lines are guide to the eye) and (b) scaled steady state velocity as a function of the concentration of dumbbells $c$. In both cases the Oldroyd-B model has been used considering $D e_{T}=3.75$.

\subsection{The effect of the Deborah number}

Figure 7a shows the steady state droplet velocity as a function of $D e_{T}$ obtained using the Oldroyd-B model for two different values of the parameter $c, c=0.5$ and $c=0.89$. The plot 
indicates that for both cases the droplet velocity decreases with $D e_{T}$. The two trends can be well approximated by a quadratic polynomial, $U / U_{Y G B} \approx 1-k_{1} D e_{T}-k_{2} D e_{T}^{2}$, with $k_{1}$ and $k_{2}$ being two constants that depend on the value of $c$. The steady state droplet shapes are illustrated in Fig. $7 \mathrm{~b}$ for different values of $D e_{T}$ and $c$.

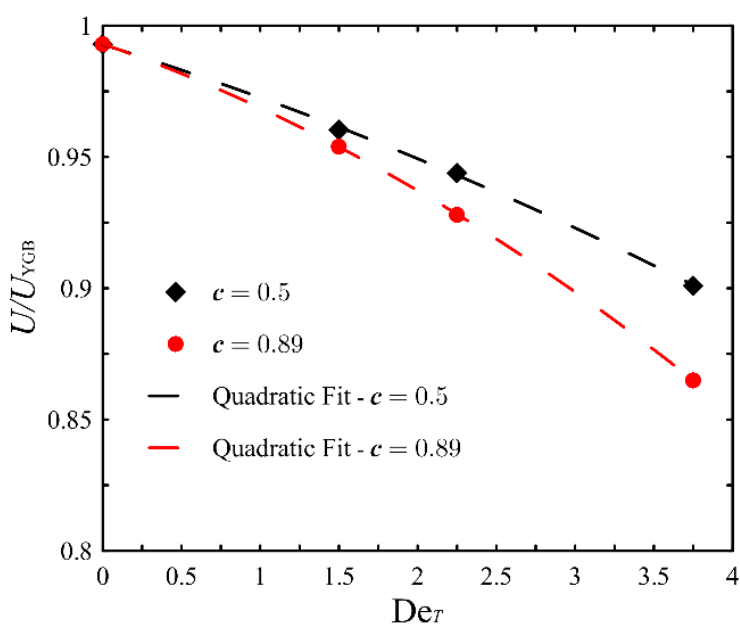

a)

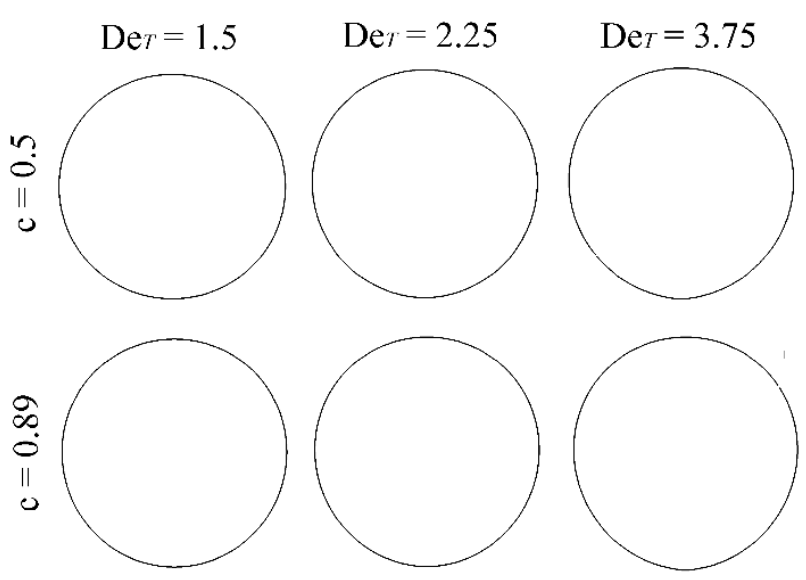

b)

Figure 7: (a) Scaled migration velocity for a droplet surrounded by the Oldroyd-B fluid as a function of the Deborah number for two values of $c$. (b) Droplet shapes for different values of the thermal Deborah for $c=0.5$ (top), and for $c=0.89$ (bottom). Note the presence of a "pointed end" for the largest values of the Deborah number.

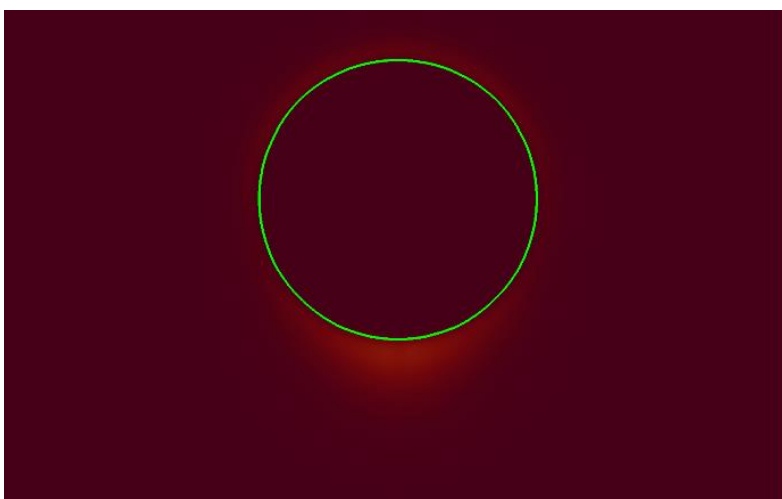

a)

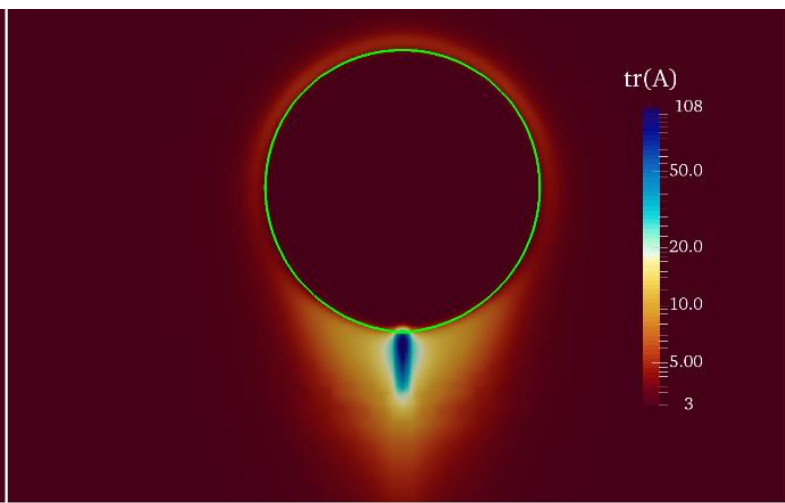

b)

Figure 8: Contours of the trace of the conformation tensor, $\mathbf{A}$, around the droplet (logarithmic scale) at steady state obtained using the Oldroyd-B model for $c=0.5$ : (a) $D e_{T}=1.5$, and (b) $D e_{T}=3.75$.

As already discussed, the droplet tends to be stretched along the direction of motion in the presence of a viscoelastic surrounding phase. For $D e_{T}=1.5$ the droplet is nearly spherical, while for the largest value of $D e_{T}$, the loss of fore-and-aft symmetry is evident, with the droplet displaying a "pointed end" (similar to the gravity-driven motion case discussed in the 
introduction) generated by the large viscoelastic stresses localised at the rear stagnation point (cf. Fig. 8 a,b). The effect of the concentration parameter on these shapes is only minimal under those conditions (even though for larger concentrations, slightly larger deformations are observed), whereas the effect of the thermal Deborah number is far more pronounced.

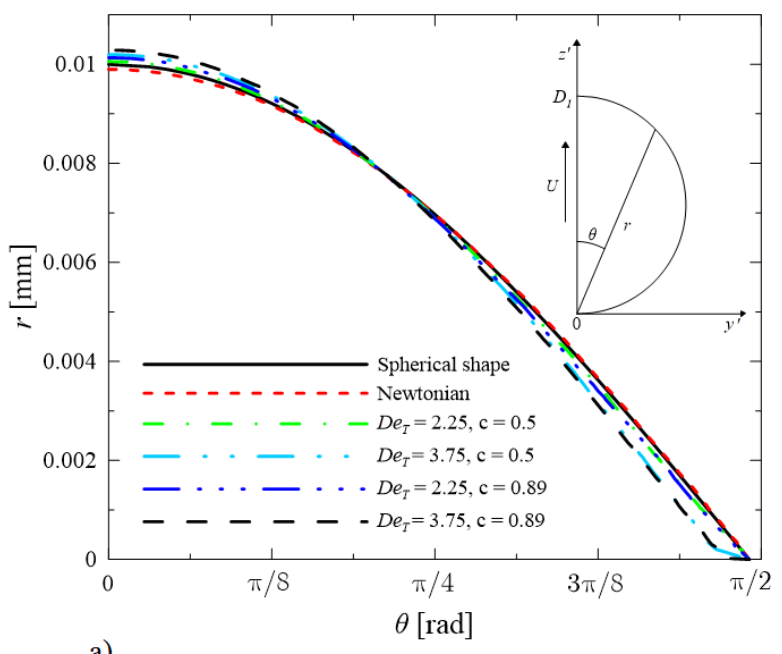

a)

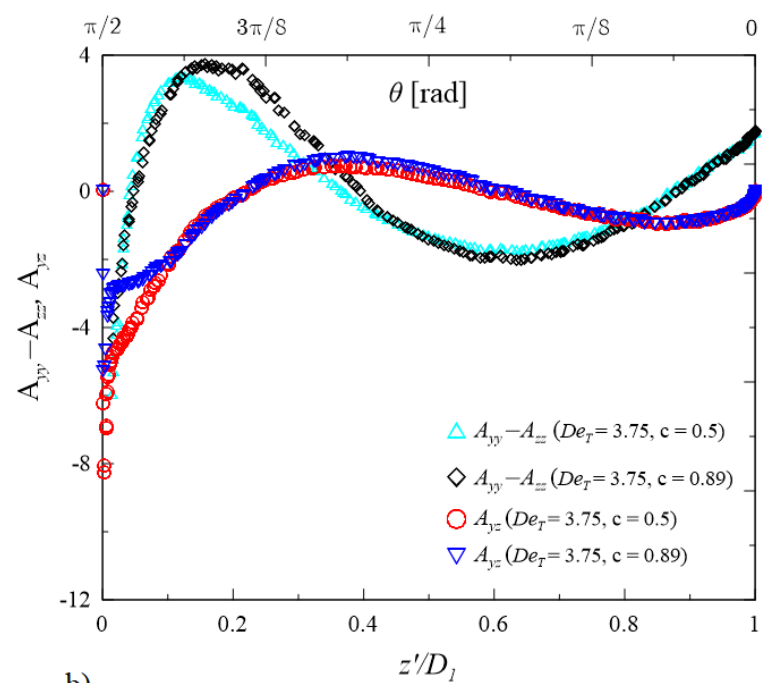

b)

Figure 9: (a) Droplet interface in a polar coordinate system attached to the drop at the rear stagnation point for different viscoelastic cases obtained using the Oldroyd-B model $(c=0.5$ and 0.89 at $D e_{T}=2.25$ and 3.75), in comparison with the Newtonian solution. For completeness, the "reference" spherical shape has been also included (continuous line). (b) Corresponding $A_{y y},-A_{z z}$ and $A_{y z}$ distribution along the reference interface for the viscoelastic simulations for $D e_{T}=3.75$ when a cusp is visible in the rear region of the drop.

To better highlight the effect of elasticity on the droplet shape, it is convenient to plot the interface in a polar coordinate system as shown in Fig. 9a (notice that in the current representation we are using absolute dimensions). This plot includes the results of our computations for the Newtonian case and various viscoelastic cases obtained with the Oldroyd-B model for different values of $c$ and $D e_{T}$. We notice that the simulated Newtonian shape (red dashed line) is nearly spherical, but a small deviation $(\sim 1 \%)$ is seen in the numerical curve resulting in a slightly oblate interface for the reasons explained in Appendix B. On the other hand, when we are in the presence of viscoelasticity, the droplets become prolate and the shapes deviate further from a sphere as $D e_{T}$ is increased. It is worth noticing that at the rear stagnation point, the interface assumes different configurations depending on the value of the Deborah number: for $D e_{T}=2.25$, the droplet is, in fact, still rounded near the rear stagnation point (cf. also Fig. 7b), and the corresponding polar plots are qualitatively similar to the Newtonian case; while for $D e_{T}=3.75$, a cusp is seen in this region (also visible in Fig. 7b). We also observe that the polar plots for all cases intersect as a direct consequence of the conservation of mass. More interestingly all viscoelastic cases intersect the corresponding Newtonian plot in the same region, which we believe is related to the distribution of the first normal stress difference (proportional to $A_{y y}-A_{z z}$ ) and viscoelastic 
shear stresses (proportional to $A_{y z}$ ) at the interface, which are shown in Fig. $9 \mathrm{~b}$ for $D e_{T}=3.75$ and two different values of $c$. We notice that regardless the value of the polymer concentration, the first normal stress difference shows a relative minimum in the region in which the intersection of the polar plots occurs (here the deformation in the $z$-direction prevails, since the value of the difference is negative), while the shear component is roughly zero.

A comparison of the flow patterns for the Newtonian flow field and some representative viscoelastic cases obtained at different values of $D e_{T}$ with the Oldroyd-B model are shown in Fig. 10. In the absence of elasticity, a large portion of the flow field is occupied by two main recirculations passing through the droplet, while a second pair of minor rolls is established next to the "cold" wall. When $D e_{T}$ is increased, the latter two recirculations tend to shrink and two new rolls become visible at the opposite "hot" wall. Finally, for the largest considered $D e_{T}$, the region covered by the new vortices embraces the whole area adjacent to the "hot" wall. 


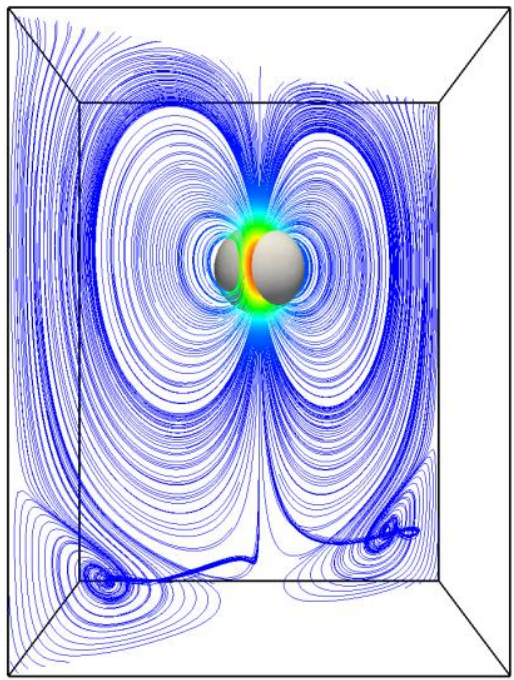

a)

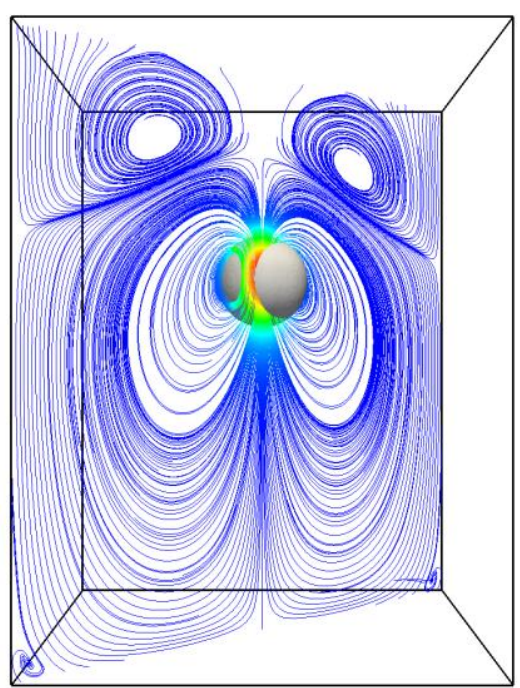

c)

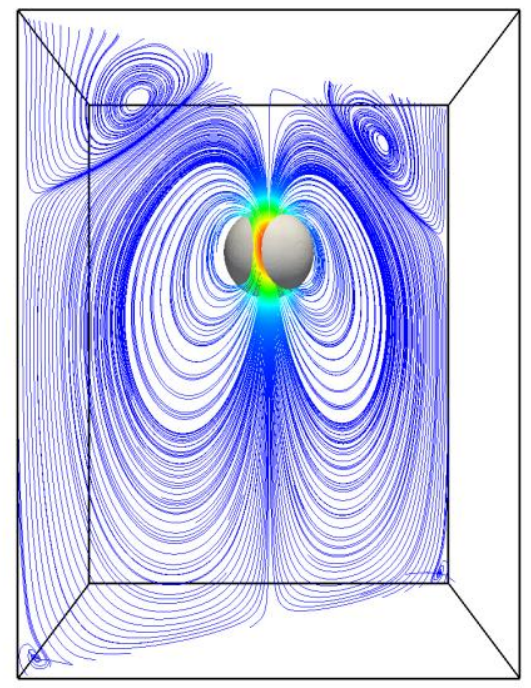

b)

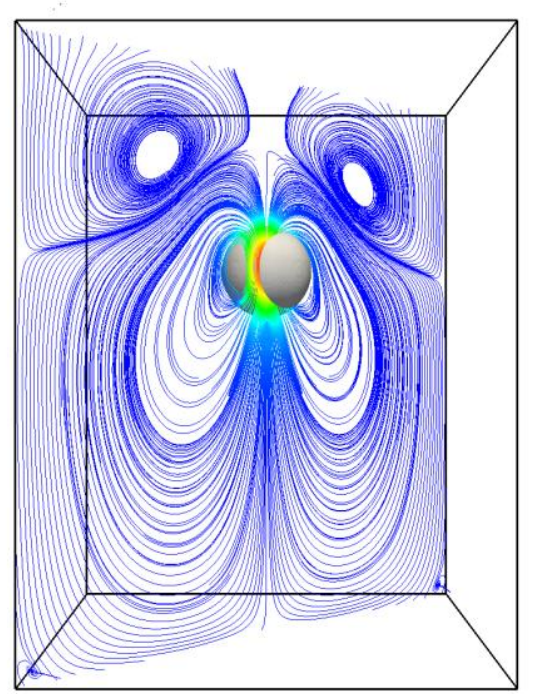

d)

Figure 10: Streamlines in a diagonal plane passing through two opposite edges of the domain under different conditions as the droplet is moving upward for Newtonian (a) and Oldroyd-B matrix fluid with b) $D e_{T}=1.5, c=0.5$, c) $D e_{T}=2.25, c=0.5$ and d) $D e_{T}=3.75, c=0.5$.

As discussed in Sect. 3.2, the Oldroyd-B model imposes severe restriction on the maximum allowable value of the Deborah number because of the singular nature of its solution when the flow field is extensional. For such reasons, the simulations shown using the Oldroyd-B model were limited to a maximum value of the Deborah number of $D e_{T}=3.75$. In order to study the impact of larger Deborah numbers, we performed a series of additional simulations on the basis of the alternative FENE-CR model. This constitutive law bounds the maximum elongation of the polymer chain through the extensibility parameter $L^{2}$, thereby allowing the 
investigation of flows at significantly higher Deborah numbers, when the Oldroyd-B model becomes unphysical.

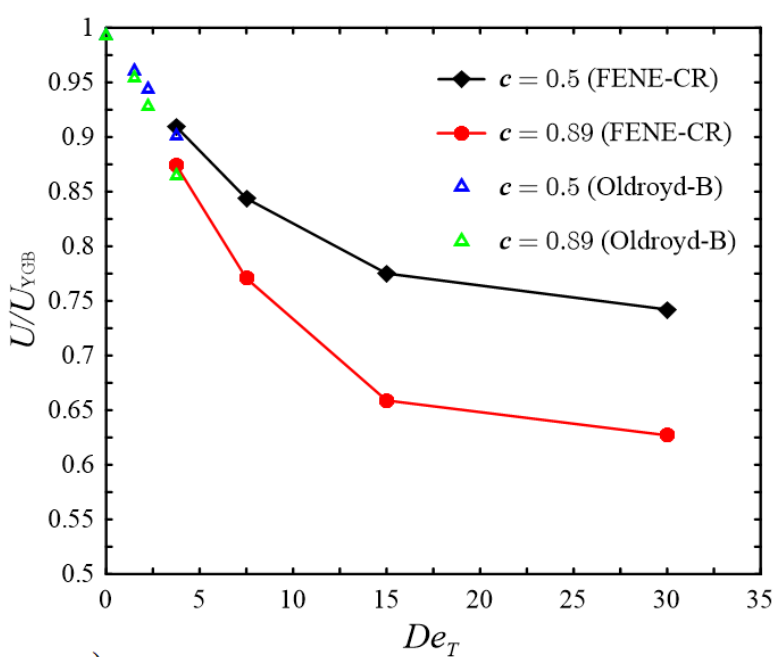

a)

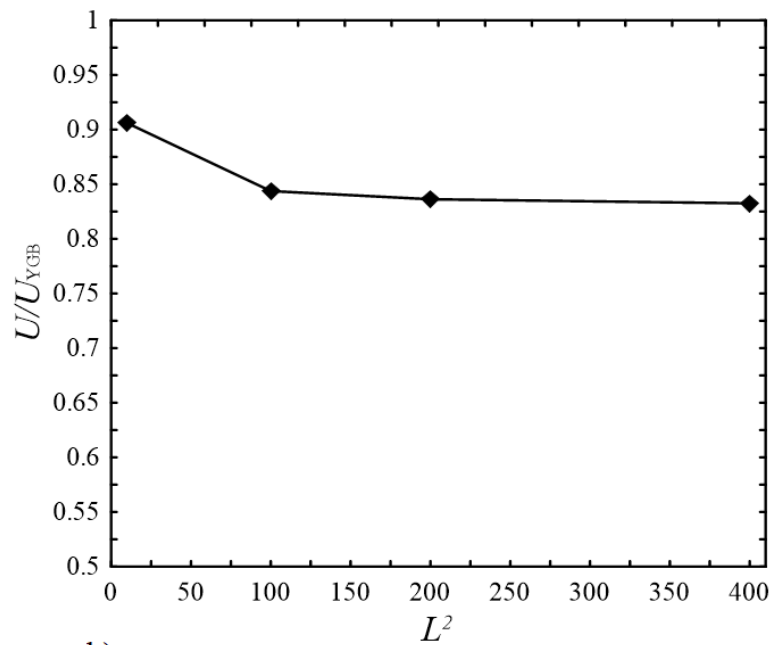

b)

Figure 11: Scaled steady state migration velocity obtained with the FENE-CR model as a function of $D e_{T}$ for two values of $c$ and $L^{2}=100$ (a), and as a function of the extensibility parameter for $D e_{T}=7.5$ and $c=0.5$ (b). The results for the Oldroyd-B case are also shown for comparison.

Fig. 11a shows the scaled migration velocity for the FENE-CR cases ( $L^{2}=100$ and two values of $c, c=0.5$ and $c=0.89$ ) as a function of $D e_{T}$. The migration velocity for the Oldroyd-B cases $\left(L^{2} \rightarrow \infty\right)$ for $D e_{T} \leq 3.75$ are also shown for comparison and it is clear that both models yield similar terminal velocities for $D e_{T}=3.75$. In fact, the relative velocity difference between these two cases is about $1 \%$, providing evidence that, for relatively small Deborah number, the maximum extensibility of the molecules does not affect the migration velocity significantly. In addition, in line with what has been observed for the case with the Oldroyd-B model at low $D e_{T}$, the steady-state droplet velocity decreases monotonically with increasing $D e_{T}$; moreover, larger values of the polymer concentrations result in smaller terminal velocities. The main qualitative dissimilarity in the trends for low $D e_{T}$ and for higher $D e_{T}$ is the different concavity of the curve, with the scaled velocity tending to a plateau region for high Deborah numbers.

In order to investigate the influence of the extensibility parameter, we conducted a series of simulations for some representative values of $L^{2}$, considering $D e_{T}=7.5$ and $c=0.5$. Figure $11 \mathrm{~b}$ shows how the terminal migration velocity decreases as the maximum allowable molecular extension is increased, tending to plateau at large values of $L^{2}$. It is also interesting to notice that for $D e_{T}=7.5$, the velocity reduction relative to the YGB limit (Young et al., ${ }^{18}$ ) 
is about $15 \%$ for $L^{2}=400$, highlighting the large impact of elasticity on the migration velocity for this range of Deborah numbers.

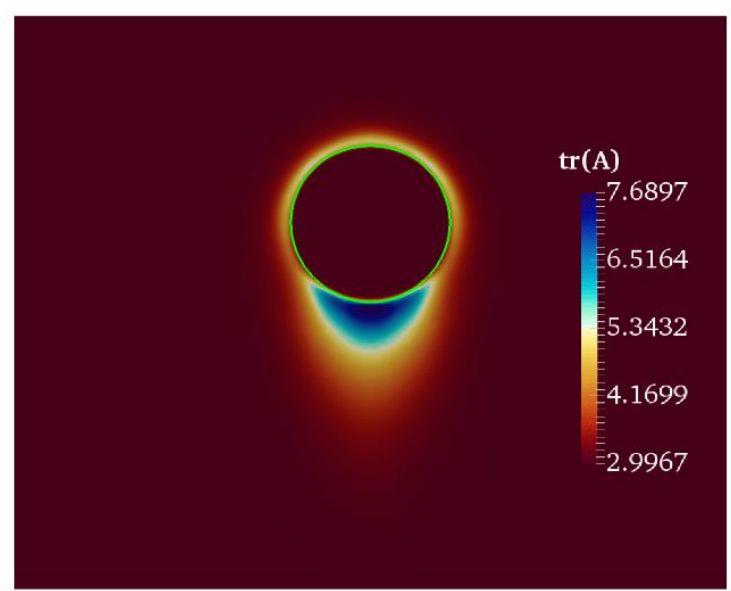

a)

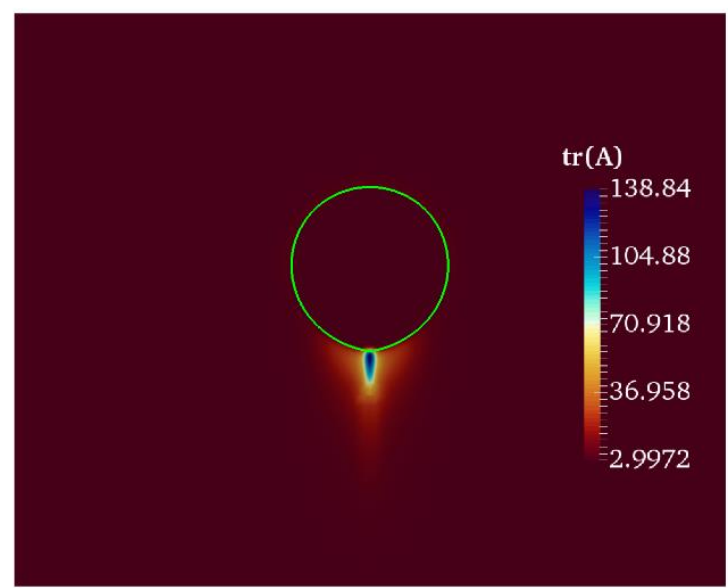

c)

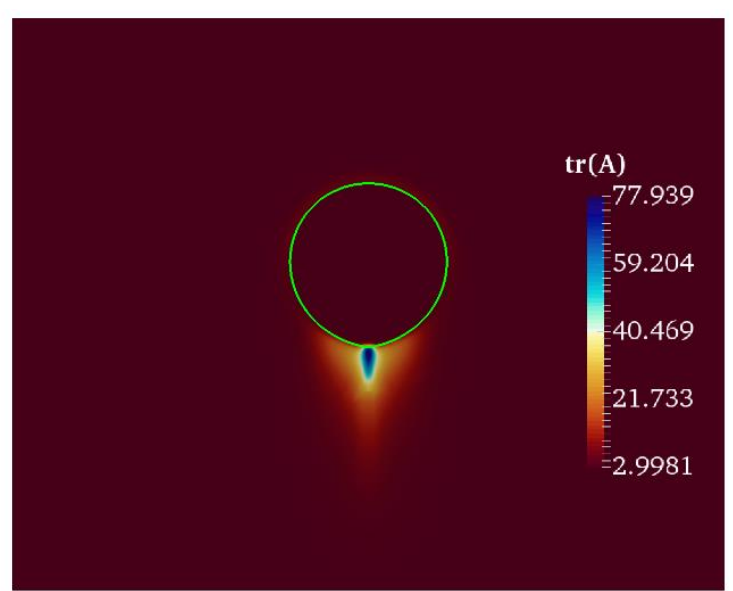

b)

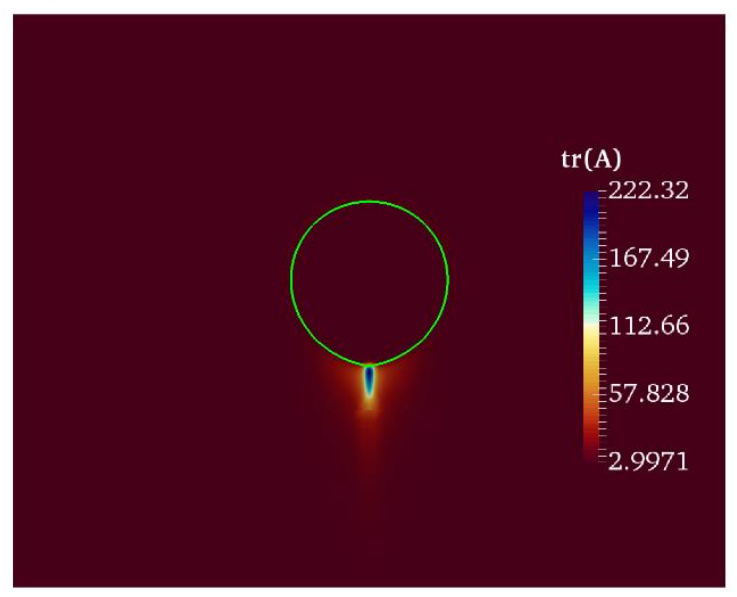

d)

Figure 12: Contours of the trace of the conformation tensor, $\mathbf{A}$, at steady state obtained using the FENE-CR model for $D e_{T}=7.5, c=0.5$ and: (a) $L^{2}=10$, (b) $L^{2}=100$, (c) $L^{2}=200$ and (d) $L^{2}=400$.

Fig. 12 shows the contours of the trace of the conformation tensor for different values of the extensibility parameter, confirming, as expected, that the normal stresses grow as the extensibility parameter $L^{2}$ is increased. It is also evident that the region of large extension, corresponding to higher values of $\operatorname{tr}(\mathbf{A})$, occupies a wider region near the rear of the droplet for small values of $L^{2} \quad\left(L^{2}=10\right.$ shown in Fig. 12a), whereas it is very localised for large values of $L^{2}$ ( $L^{2}=100,200$ and 400 shown in Fig. 12b-d). These localised stresses will arguably have a direct impact on the deformation of the droplet surface and the formation of the cusp as shown in Fig. 13, where we plot the droplet interface in a polar coordinate system (akin to that used in Fig. 9) to highlight the differences in droplet shape for varying $L^{2}$. Notice 
that the shape for the three largest values of $L^{2}$ studied is very similar, exhibiting a cusp near the rear stagnation point $(\theta=\pi / 2)$, while this cusp is absent for $L^{2}=10$.

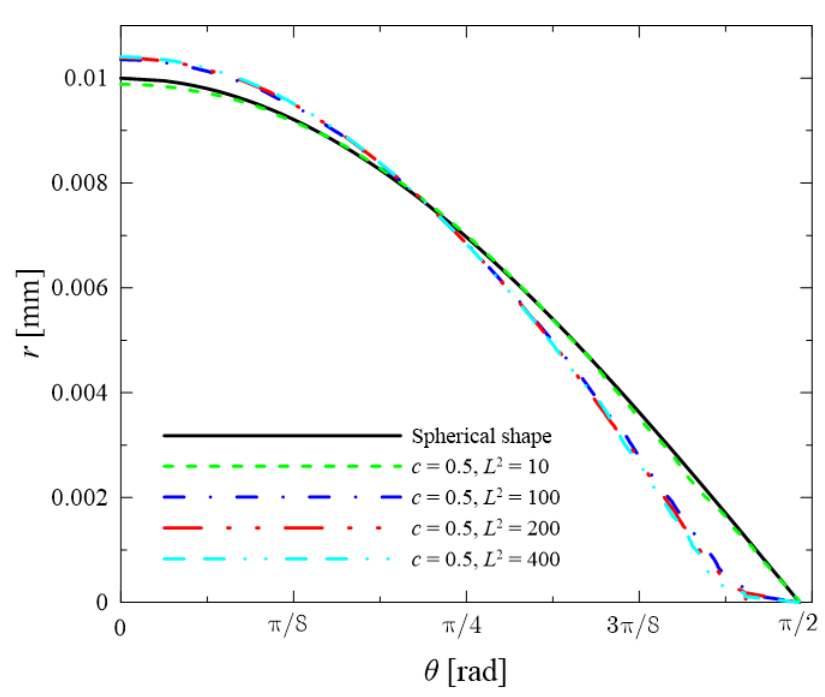

Figure 13: Droplet interface in a polar coordinate system attached to the drop at the rear stagnation point obtained using the FENE-CR model for $D e_{T}=7.5, c=0.5$ and: (a) $L^{2}=10$, (b) $L^{2}=100$, (c) $L^{2}=200$ and (d) $L^{2}=400$. The spherical reference shape (continuous black line) has been added for comparison.

Additional insights can be gathered from Fig. 14, which shows the droplet shape evolution for the cases $c=0.5$ (a) and $c=0.89$ (b) for $D e_{T}=30$ and $L^{2}=100$. Initially (instant $t_{1}$ ), the drop does not display a significant deformation, and its shape is a prolate ellipsoid. As time passes (instant $t_{2}$ ), the viscoelastic stresses, which are mainly developing around the rear of the droplet (as already discussed for the cases with the Oldroyd-B model), lead to fore-and-aft symmetry breaking (though the pointed end is not yet visible). In particular, at this stage the rear of the drop is more flattened for the case $c=0.89$ than for the case for $c=0.5$, indicating that during the transient the viscoelastic stresses tend to be distributed differently depending on the value of the parameter $c$. At the instant $t_{3}$, for $c=0.5$ the presence of a pointed end can be noticed, which is not yet visible for the higher concentration $c=0.89$. Finally, at the last stage (instant $t_{4}$ ) the presence of the pointed end can also be clearly noticed for the larger value of $c$. Interestingly, even though the terminal velocity is larger for smaller values of $c$, between the instants $t_{1}$ and $t_{2}$ the droplet has travelled for a longer distance for $c=0.89$ rather than in the case $c=0.5$. Such a difference has to be ascribed to the well-known fact that the viscoelastic stresses require a certain amount of time to develop. Initially, the contribution to the hydrodynamic resistance is mainly due to the presence of viscous stresses. As these stresses are proportional to the solvent viscosity, $\eta_{s, m}$, and since for $c=0.89, \eta_{s, m}$ is lower than that for $c=0.5$, the velocity is initially larger. 
We conclude that independently of the Deborah number, polymer concentration and extensibility parameter, the flow patterns established in the first half of the drop seem to be qualitativley similar. On the contrary, in the rear part of the drop, the differences are much more pronounced, and might be attributed to memory effects that become more prominent as the polymer molecules travel around the drop.

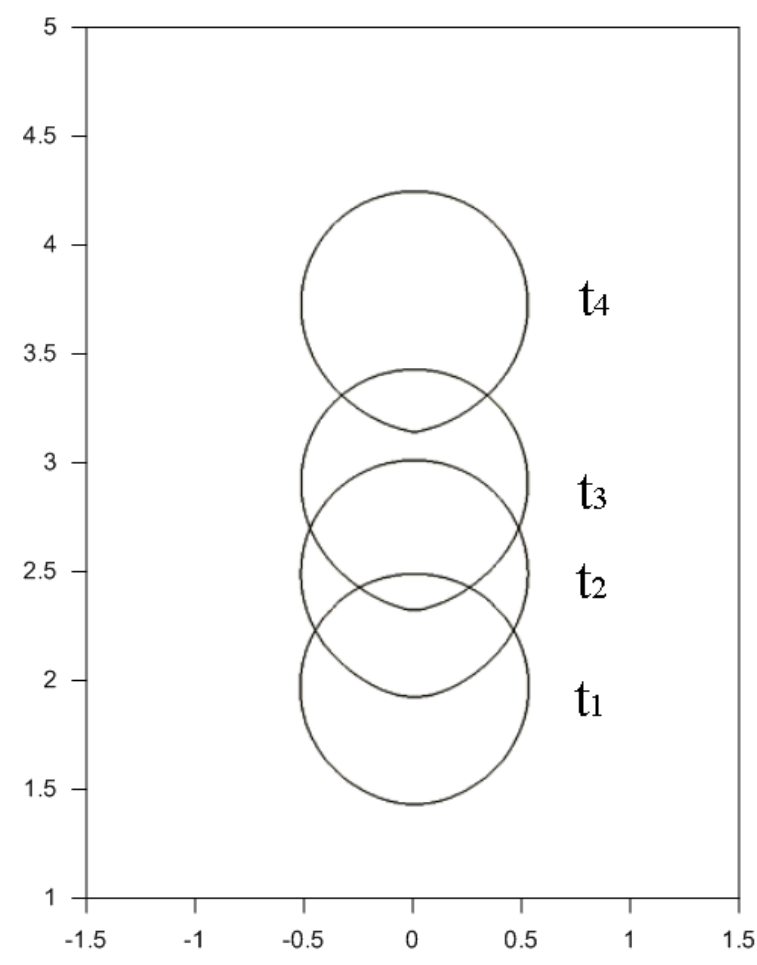

a)

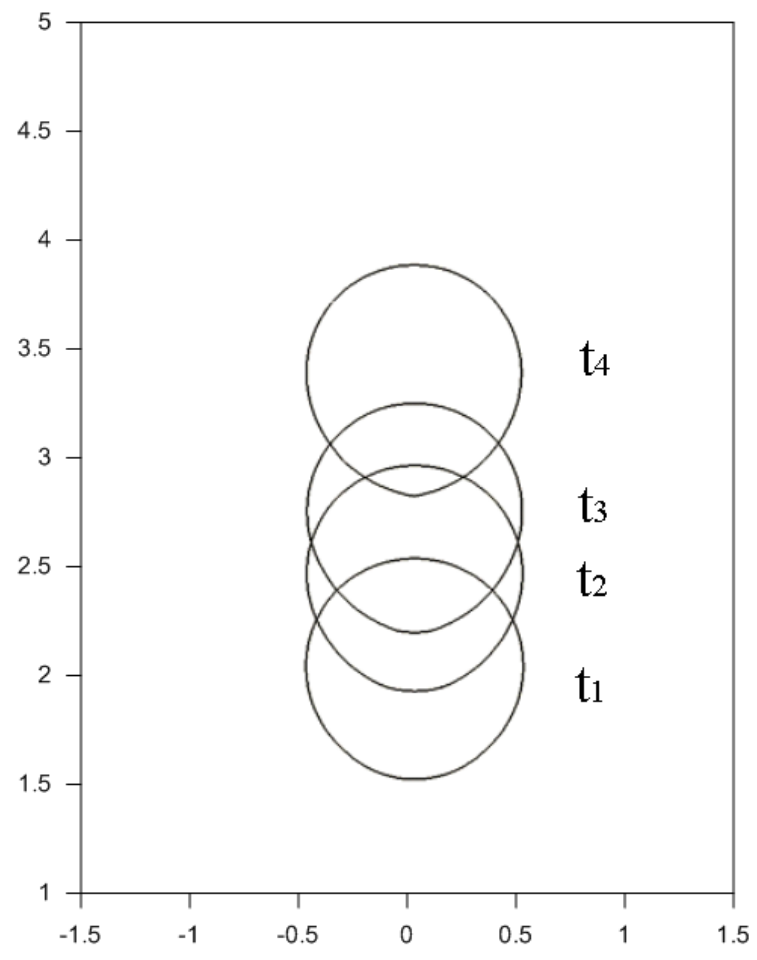

b)

Figure 14: Droplet shape temporal evolution obtained using the FENE-CR model for $D e_{T}=30$ and $L^{2}=100$, for $c=0.5$ (a), and for $c=0.89$ (b). The time frames are the same for the two pictures, evidencing the different droplet transient velocity evolution.

\section{Conclusions}

The thermocapillary motion of a Newtonian deformable droplet surrounded by a viscoelastic immiscible liquid has been investigated numerically over a relatively wide range of conditions. The impact of viscoelasticity on the droplet morphology and migration mechanism has been assessed in the framework of two viscoelastic constitutive laws. In particular, the classical Oldroyd-B model, used for relatively small values of the thermal Deborah number (due to its simplicity and widespread success in the literature), has been replaced by the more stable and realistic FENE-CR model (in order to circumvent the typical 
unphysical singularities that develop for such conditions in the equations governing fluid flow when using the Oldroyd-B model) in this way higher values of $D e_{T}$ have been attained, up to a maximum value of 30 . In addition, two distinct flow conditions have been addressed, namely the case of an "infinitely dilute" solution, expressly considered to analyse the deformation history of polymer molecules flowing in a Newtonian flow field (i.e., in absence of viscoelastic stresses), and the case of a finite small dilution, where the coupling between the viscoelastic stresses and the flow field is expected to modify such a process and the extent of the deformation of the molecules (as they flow around the drop).

The numerical experiments show that large viscoelastic stresses tend to be concentrated in proximity to the rear stagnation point, where owing to the extensional nature of the flow the largest polymer molecules deformation is attained. The value of the parameter $c$ has a strong impact on the maximum dumbbell elongation, which decreases for increasing values of the concentration. For finite values of $c$, it has a remarkable influence on the viscoelastic stresses and, as a natural consequence, on both the migration velocity (higher droplet migration velocities are seen for lower concentrations) and droplet shape.

In terms of the effect of Deborah number, the migration velocity of the droplet has been found to be a monotonic decreasing function of $D e_{T}$ for the range of conditions considered. With regard to the droplet morphological evolution, the droplet initially becomes a prolate ellipsoid and then a certain degree of loss of fore-and-aft symmetry develops as the Deborah number increases. Specifically, for the largest values of the thermal Deborah number, the concentration of viscoelastic stresses near the rear stagnation point has been found to be responsible for the development of a "pointed tail".

Finally, the effect of the extensibility parameter on droplet dynamics has been investigated for some selected cases. The results show that the related impact in terms of the steady state droplet speed and shape (when compared to the Newtonian case) is more pronounced for larger values of $L^{2}$, for which the normal stresses are larger and more localised near the rear stagnation point, than for small values of $L^{2}$. For large values of $L^{2}\left(L^{2}>100\right)$, the droplet shape and speed become nearly independent of the value of $L^{2}$.

\section{Acknowledgements}

Results were obtained using the ARCHIE-WeST High performance Computer (www.archie.west.ac.uk) based at the University of Strathclyde. The authors would also like to thank Professor Rob Poole and Mr Mahdi Davoodi from the University of Liverpool for the useful discussions and suggestions. 


\section{References}

[1] Rotheiser, J., Joining of Plastics (Hanser, 1999).

[2] Troughton, M. J., Handbook of Plastics Joining: A Practical Guide (William Andrew, 2008).

[3] Aly, A.A., (2015), Heat Treatment of Polymers: A Review, International Journal of Materials Chemistry and Physics, 1(2), 132-140.

[4] Grewell, D. and Benatar, A., (2007), Welding of plastics: Fundamentals and new developments, Int. Polym. Process. 22(1), 43-60.

[5] Karbalaei, A., Rangananthan, K. and Jin Cho, H. (2016) Thermocapillarity in microfluidics-A review. Micromachines. 7, 13.

[6] Chhabra, R. P., (2007), Bubbles, Drops, and Particles in Non-Newtonian Fluids, second edition, Taylor \& Francis, London.

[7] Bonito, A., Clément, P. and Picasso M., (2011), Viscoelastic Flows with Complex Free Surfaces: Numerical Analysis and Simulation, pp. 305-370 In Handbook of numerical analysis, Special Volume (Vol. XVI): Numerical Methods for Non-Newtonian Fluids, Guest Editors: R. Glowinski and J. Xu, Nort-Holland (Elsevier, 2011).

[8] Dee, G. T. and Sauer, B. B., (1998), The surface tension of polymer liquids, Adv. Phys. 47(2), 161-205.

[9] Philippoff, W. (1937), The viscosity characteristics of rubber solutions, Rubber Chemistry and Technology 10, 76-104.

[10] Warshay, M., Bogusz, E., Johnson, M., and Kintner, R.C., (1959), Ultimate velocity of drops in stationary liquid media, Can. J. Chem. Eng., 37, 29-36.

[11] Mhatre, M. V. and Kintner, R. C., (1959), Fall of liquid drops through pseudoplastic liquids, Ind. Eng. Chem. 57, 865-867.

[12] Astarita G and Apuzzo G., (1965), Motion of gas bubbles in non-Newtonian liquid, AIChE J., 11, 815-820.

[13] Calderbank, P.H., (1967), Gas absorption from bubbles, Chem. Eng., 45, 209 p.

[14] Calderbank, P.H., Johnson, D.S.L. and Loudon, J., (1970), Mechanics and mass transfer of single bubbles in free rise through some Newtonian and non-Newtonian liquids, Chem. Eng. Sci., 25, 235-256.

[15] Zana, E. and Leal, L.G., (1978), The dynamics and dissolution of gas bubbles in a viscoelastic fluid, Int. J. Multiphase Flow, 4, 237-262.

[16] Hassager, O., (1979), Negative wake behind bubbles in non-Newtonian liquids, Nature, 279, 402.

[17] Liu Y. J., Liao, T. Y. and Joseph, D. D., (1995), A two-dimensional cusp at the trailing edge of an air bubble rising in a viscoelastic liquid, J. Fluid Mech., 304, 321-342

[18] Young, N.O., Goldstein, J.S. and Block M.J., (1959), The motion of bubbles in a vertical temperature gradient, J. Fluid Mech, 6, 350-360.

[19] Betz, J. and Straub, J., (2002), Thermocapillary convection around gas bubbles: An important natural effect for the enhancement of heat transfer in liquids under microgravity, Annals of the New York Academy of Sciences, 220-45.

[20] Villers, D. and Platten, J. K., (1987), Separation of Marangoni convection from gravitational convection in earth experiments. PhysicoChem., p. 173-183. 
[21] O'Shaughnessy, S. M. and Robinson, A., (2013), Heat Transfer near an Isolated Hemispherical Gas Bubble: The Combined Influence of Thermocapillarity and Buoyancy, International Journal of Heat and Mass Transfer, 422-434.

[22] Hadland, P.H., Balasubramaniam, R., Wozniak, G. and Subramanian R.S., (1999), Thermocapillary migration of bubbles and drops at moderate to large Marangoni number and moderate Reynolds number in reduced gravity, Exp. Fluids, 26: 240-248.

[23] Subramanian, R.S., (1983), "Thermocapillary migration of bubbles and droplets", $A d v$. Space Res., 3(5): 145-153.

[24] Balasubramaniam, R. and Chai A.-T., (1987), "Thermocapillary migration of droplets: An exact solution for small Marangoni numbers", J. Colloid Interface Sci., 119(2): 531-538.

[25] Balasubramaniam, R. and Subramanian R. S, (2000), The migration of a drop in a uniform temperature gradient at large Marangoni numbers, Phys. Fluids, 12(4): 733-743.

[26] Haj-Hariri, H., Shi Q. and Borhan A., (1997), Thermocapillary motion of deformable drops at finite Reynolds and Marangoni numbers, Phys. Fluids 9 (4):845-855.

[27] Rudman, M., (1998), Volume tracking methods for interfacial flow calculations, International Journal for Numerical Methods in Fluids, 24, 671-691.

[28] Gueyffier, D., Li, J. , Nadim, A., Scardovelli S. and Zaleski S., (1999), "Volume of Fluid interface tracking with smoothed surface stress methods for three-dimensional flows", $J$. Comput. Phys., 152: 423-456

[29] Sussman, M. and Fatemi, E. (1999): An efficient, interface-Preserving Level Set Redistancing Algorithm and its application to Interfacial Incompressible Fluid Flow. SIAM J. Sci. Comput., 20, 1165-1191.

[30] Sussman, M. and Puckett, E. (2000), A coupled level set and volume-of-fluid method for computing 3D and axisymmetric incompressible two-phase flows. J. Comput. Phys., 162, 301-337.

[31] Liu, H., Wu, L., Ba, Y. and Xi, G., (2017), A lattice Boltzmann method for axisymmetric thermocapillary flows, International Journal of Heat and Mass Transfer 104, 337-350.

[32] Liu, H. and Zhang, Y., (2015), Modelling thermocapillary migration of a microfluidic droplet on a solid surface, Journal of Computational Physics, 280, 37-53 (2015).

[33] Guo, Z. and Lin, P., (2015), A thermodynamically consistent phase-field model for twophase flows with thermocapillary effects, J. Fluid Mech., 766, 226-271.

[34] Liu, H., Valocchi, A. J., Zhang, Y. and Kang, Q., (2013), Phase-field based lattice Boltzmann finite difference model for simulating thermocapillary flows. Physical Review E, 87, 013010.

[35 ]Getachew, D. and Rosenblat, S., (1985), Thermocapillary instability of a viscoelastic liquid layer, Acta Mechanica, 55, 137-149.

[36] Martinez-Mardones, J. and Perez-Garci, C., (1990), Linear instability in viscoelastic fluid convection, Journal of Physics: Condensed Matter, 2, 5.

[37] Parmentier, P., Lebon, G. and Regnie, V., (2000), Weakly nonlinear analysis of BénardMarangoni instability in viscoelastic fluids, Journal of Non-Newtonian Fluid Mechanics, 89, 63-95. 
[38] Hu, K., He, M., Chen, Q., and Liu, R., (2018), Effect of gravity on the stability of viscoelastic thermocapillary liquid layer, International Journal of Heat and Mass Transfer, 123, 776-786.

[39] Lappa, M. and Ferialdi, H., (2018), Multiple solutions, Oscillons and Strange Attractors in ThermoViscoElastic Marangoni Convection, Phys. Fluids, 30(10), 104104 (19 pages).

[40] Jiménez-Fernández, J. and Crespo, (2002), The thermocapillary migration of gas bubbles in a viscoelastic fluid. Microgravity Sci. Technol., 13, 33.

[41] Capobianchi, P., Lappa, M. and Oliveira, M.S. N., (2017), Walls and Domain Shape Effects on the Thermal Marangoni Migration of Three-dimensional Droplets, Phys. Fluids 29, 112102.

[42] Capobianchi, P., Lappa, M. and Oliveira, M.S. N., (2019), Implementation of a Flexible and Modular Multiphase Framework for the Analysis of Surface-Tension-Driven Flows Based on a LS-VOF Approach, in Selected papers of the 11th OpenFOAM Workshop, J.M.Nóbrega and H. Jasak (Editors), Springer Nature, ISBN - 978-3-319-60845-7.

[43] Tryggvason, G., Bunner, B., Esmaeeli, A., Juric, D., Al-Rawahi, N., Tauber, W., Han, J., Nas, S.,and Jan Y.-J., (2001), A Front Tracking Method for the Computations of Multiphase Flow, J. Comput. Phys., 169: 708-759.

[44] Oldroyd, J. G. (1950). On the Formulation of Rheological Equations of State. Proc. R. Soc. Lond. A. 200, 523-541.

[45] Chilcott, M. D. and Rallison, J. M., (1988) Creeping flow of dilute polymer solutions past cylinders and spheres. J Non-Newton Fluid Mech, 29, 381-432.

[46] Bird, R. B., Curtiss, C.F., Armstrong, R. C. and Hassager, O., (1987), Dynamics of Polymeric Liquids, vol. 2, 2nd edn. John Wiley \& Sons, New York.

[47] Alves, M.A., Oliveira, P.J. and Pinho, F.T., (2003), Benchmark solutions for the flow of Oldroyd-B and PTT fluids in planar contractions, J. Non-Newtonian Fluid Mech., 110, 4575.

[48] Du, Q., Liu, C. and Yu, P. (2005). FENE dumbbell model and its several linear and nonlinear closure approximations, Multiscale Model, Simul. 4 (3), 709-731.

[49] Cherizol, R., Sain, M. and Tjong, J. (2015) Review of Non-Newtonian Mathematical Models for Rheological Characteristics of Viscoelastic Composites, Green and Sustainable Chemistry, 5, 6-14.

[50] Kramers, H. A.(1946) The behaviour of macromolecules in inhomogeneous flow, J. Chem. Phys. 14, 415-424.

[51] Capobianchi, P., Lappa, M. and Oliveira, M.S. N., (2018), Deformation of a Ferrofluid Droplet in a Simple Shear Flow Under the Effect of a Constant Magnetic field, Computers \& Fluids, 173. pp. 313-323.

[52] Yamamoto, T., Okano, Y. and Dost, S., (2016), Validation of the S-CLSVOF method with the density-scaled balanced continuum surface force model in multiphase systems coupled with thermocapillary flows, International Journal of Numerical Methods in Fluids, 83, 223-244.

[53] Albadawi, A., Donoghue, D. B., Robinson, A. J., Murray, D. B. and Delauré, Y. M. C., (2013), Influence of surface tension implementation in volume of fluid and coupled Volume of Fluid with Level Set methods for bubble growth and detachment, International Journal of Multiphase Flow, 53, 11-28. 
[54] Moukalled, F., Mangani, L. and Darwish M., (2016), The Finite Volume Method in Computational Fluid Dynamics - An Advanced Introduction with OpenFOAM and Matlab, Springer International Publishing, 2016, New York).

[55] Tryggvason, G., Scardovelli, R. and Zaleski S., (2011), Direct numerical simulations of gas-liquid multiphase flows, Cambridge University Press.

[56] Sussman M., Smereka P., Osher S., (1994), A Level Set Approach for Computing Solutions to Incompressible Two-Phase Flow, J. Comput, Physics, 114(1),146-159.

[57] Son, G. and Hur, N., 2002. A coupled level set and volume-of-fluid method for the buoyancy-driven motion of fluid particles. Numer. Heat Transfer, Part B: Fundam. 42, 523542.

[58] Sun, D. and Tao, W., 2010. A coupled volume-of-fluid and level set (voset) method for computing incompressible two-phase flows. Int. J. Heat Mass Transfer 53, 645-655.

[59] Favero, J. L., Cardozo, N. S., Secchi, A. R. and Jasak, H., (2010), Simulation of Free Surface Viscoelastic Fluid Flow Using the viscoelasticInterFoam Solver," in 20th European Symposium on Computer Aided Process Engineering, pp. 31-36.

[60] Pillapakkam, S. B. and Singh, P., (2001), A Level-Set Method for Computing Solutions to Viscoelastic Two-Phase Flow, Journal of Computational Physics, 174, 2, 552-578.

[61] Chinyoka, T., Renardy, Y., Renardy, M. and Khismatullin, D.B., (2005), Twodimensional study of drop deformation under simple shear for Oldroyd-B liquids, J. NonNewtonian Fluid Mech., 130, 45-56.

[62] Renardy, M. (1999), High Weissenberg Number Asymptotics and Corner Singularities in Viscoelastic Flows. In: Durban D., Pearson J.R.A. (eds) IUTAM Symposium on Nonlinear Singularities in Deformation and Flow. Springer, Dordrecht

[63] Owens R. G. and Phillips T. N., (2002), Computational Rheology, Imperial College Press, 2002, London.

[64] Siginer D., 2014), Stability of Non-Linear Constitutive Formulations for Viscoelastic Fluids, Springer Briefs in Thermal Engineering and Applied Science (2014, Springer International Publishing, New York).

[65] Harlen, O. G., (2005), The negative wake behind a sphere sedimenting through a viscoelastic fluid, J. Non-Newtonian Fluid Mech., 108, 411-430.

[66] Pillapakkam, S. B., Singh P., Blackmore, D. and Aubry, N., (2007), Transient and steady state of a rising bubble in a viscoelastic fluid, J. Fluid Mech., 589, 215-252.

[67] Rhie, M. C. and Chow, L. W., (1982), A numerical study of the turbulent fow past an isolated airfoil with trailing edge separation, AIAA-82-0998.

[68] Deshpande S. S., Anumolu L., Trujillo, M. F., (2012), Evaluating the performance of the two-phase flow solver interFoam, Computational Science and Discovery, 5014016.

[69] Ling, Y., Fullana, J., Popinet, S. and Josserand, C., (2016), Droplet migration in a HeleShaw cell: Effect of the lubrication film on the droplet dynamics. Phys. Fluids 28, 062001. 


\section{APPENDIX A: Algorithm description}

The governing equations are solved in a structured Cartesian grid using a Finite Volume Method (FVM) relying on their integral formulation over a set of control volumes. All the variables are stored at the centre of cells, however the solution methodology employed by OpenFOAM involves also their values interpolated at the cell face. In order to avoid unphysical oscillation (checkerboard effect) due to the non-staggered collocation of the variables, the Rhie-Chow ${ }^{67}$ interpolation is used.

The solution of the entire set of equations can be summarised as follows:

1. Set the boundary and initial conditions;

2. Solve the re-initialisation equation (11) to calculate the level-set function $\varphi_{k}$;

3. Solve the diffusion equation (12) to obtain the smoothed level-set function $\varphi_{k, \text { mol }}$;

4. Calculate the interface normal and curvature by means of Eqs. 13 and14;

5. Advect the volume fraction, $\alpha_{k}$, by means of Eq. 14 using the MULES algorithm (Multidimensional Universal Limiter with Explicit Solution) (see, e.g.,Deshpande et al. $\left.{ }^{68}\right)$. Applying Gauss' theorem, the integration of Eq. (14) leads to

$\int_{\Gamma_{c . i}} \frac{\partial \alpha_{k}}{\partial t} d V+\int_{\partial \Gamma_{c . i}}\left(\alpha_{k} \mathbf{u}+\alpha_{k}\left(1-\alpha_{k}\right) \mathbf{u}_{\mathrm{c}}\right) \cdot \mathrm{n} d S=0$

where $\Gamma_{c, i}$ is the volume of the computation cell $i$ and $\partial \Gamma_{c, i}$ its boundary. Using the forward Euler scheme, the discrete counterpart of Eq. (22) can be written as

$\left|\Gamma_{c, i}\right| \frac{\alpha_{c, i}^{n+1}-\alpha_{c, i}^{n}}{\Delta t}=-\sum_{f_{c, i}} F_{u}^{n}-\sum_{f_{c, i}} \zeta_{M} F_{c}^{n}$

where the flux term $F_{u}$ arises from the integration of $\nabla \cdot\left(\alpha_{k} \mathbf{u}\right)$, and the term $F_{c}$ is a linear combination of the flux associated with the integration of the compressive term $\nabla \cdot\left(\alpha_{k}\left(1-\alpha_{k}\right) \mathbf{u}_{\mathrm{c}}\right)=0$ and the previous flux $F_{u}$ (see Deshpande et al. ${ }^{68}$ for more details). The coefficient $\zeta_{M}$ appearing in the second term on the right-hand-side of Eq. (18) is the MULES limiter. The term $F_{c}$ is active only across the interface, where $\zeta_{M}=1$, whereas $\zeta_{M}=0$ away from the interface, which makes $F_{c}$ inactive. The limiter therefore splits the numerical treatment of the advection term into two parts: away from the interface, the second summation appearing in Eq. (23) is set to zero, and $F_{u}$ is treated with an upwind scheme, while across the interface, where a better accuracy is required, a higher order scheme is employed. This strategy allows to reduce the computational effort by activating the more accurate scheme only in the region of the interface, where higher accuracy is required. Finally, the compressive velocity $\mathbf{u}_{c}$ defined previously takes the following form 
$\mathbf{u}_{c}=\min \left[C_{\alpha} \frac{\left|\mathbf{u}_{f} \cdot \mathbf{S}_{f}\right|}{\left|\mathbf{S}_{f}\right|}, \max \left(\frac{\left|\mathbf{u}_{f} \cdot \mathbf{S}_{f}\right|}{\left|\mathbf{S}_{f}\right|}\right)\right] \mathbf{n}_{f}$

Here, $\mathbf{u}_{f}, \mathbf{S}_{f}$ and $\mathbf{n}_{f}$ are the velocity vector interpolated at the cell face, cell face vector and cell face normal, respectively. The numeric constant constant, $C_{\alpha}$, is a user defined parameter and usually is set in the range 0 (the compressive velocity is inactive) to 2. Larger values of $C_{\alpha}$ correspond to a sharper interface but higher spurious currents. In our simulations we used $C_{\alpha}=2$ for all cases.

6. Solve the energy Eq. 4 to get the temperature field. This equation was implemented in our method in a slightly different form, as discussed in detail by Capobianchi et al. ${ }^{42}$ that was proved to be more stable, particularly for problems at large Marangoni numbers. Recalling the definition of the thermal diffusivity, $\alpha_{t h}=\kappa / \rho c_{p}$, after some manipulations the energy equation can be written as:

$\frac{D T}{D t}=\nabla \cdot\left(\alpha_{t h} \nabla T\right)+\frac{1}{\rho c_{p}} \nabla \kappa \cdot \nabla T-\nabla \alpha_{t h} \cdot \nabla T$

The first term on the right hand side is treated implicitly, while the gradients appearing on the other two terms are treated explicitly;

7. Calculate the thermocapillary force, $\mathbf{f}_{\sigma, \mathrm{t}}=\sigma_{T}\left(\mathbf{I}-\mathbf{n}\left(\varphi_{k, \text { mol }}\right) \mathbf{n}\left(\varphi_{k, \text { mol }}\right)\right) \nabla T\left|\nabla \alpha_{k}\right|$;

8. Solve for the viscoelastic model Eq. (6);

9. Calculate of the elastic stress tensor $\tau$ by means of Eq. (8);

10. Evaluate the divergence of the total stress tensor: $\nabla \cdot \Sigma=\nabla \cdot\left(2 \eta_{\mathrm{s}} \mathbf{D}+\tau\right)$. This term is treated into the code in the following form

$\nabla \cdot \Sigma=\nabla \cdot\left(\left(\eta_{\mathrm{s}}+\eta_{p}\right) \nabla \mathbf{u}\right)-\nabla \cdot\left(\eta_{p} \nabla \mathbf{u}\right)+\nabla \mathbf{u} \cdot \nabla \eta_{\mathrm{s}}+\nabla \cdot \tau$

where all the terms on the right hand side are treated explicitly, with the exception of the first term, which is treated implicitly. Note the addition and subtraction of $\nabla \cdot\left(\eta_{p} \nabla \mathbf{u}\right)$. The presence of this extra diffusive term serves to stabilise the solution of the momentum equation;

11. Solve the momentum Eq. 15 for a prefixed number of predictor steps to get an initial value of the velocity field;

12. Perform the PISO loop to calculate pressure and the velocity fields until momentum and mass conservation are both satisfied;

13. Check convergence criterion and go back to step 2 or end of calculation.

\section{APPENDIX B: Grid refinement analysis}

The outcomes of a mesh-refinement study are described here. Following common practice in the literature (see, e.g., Ling et al. ${ }^{69}$ ), in order to save computational time we performed the 
assessment in 2D under the assumption that, for the considered category of problems, the same level of grid refinement in the 3D space will lead to the same level of grid independence. In particular, the effect of the grid spacing was assessed for three different mesh resolutions, namely $M_{1}, M_{2}$ and $M_{3}$ (as indicated in Table II) considering the both the Newtonian and viscoelastic configurations ( adopting the Oldroyd-B model considering $D e_{T}$ $=3.75, \beta=0.5$ ) and setting the maximum Courant number at $C o_{\max }=0.02$. The time step is already very restrictive, but this was deemed necessary to guarantee acceptable droplet shapes (as discussed below). The results summarised in Table II show the good convergence in terms of migration velocity and the relative difference between the cases $M_{1}$ and $M_{3}$ for all cases considered.

Table II Characteristics of the 2D meshes used for the mesh-independence assessment study. The results are shown in terms of velocity at $t^{\prime}=40$ for three different cases and the velocity difference is evaluated relative to the case $M_{3}$.

\begin{tabular}{|c|c|c|c|}
\hline & $M_{1}$ & $M_{2}$ & $M_{3}$ \\
\hline $\mathrm{N}^{\circ}$ of cells per droplet diameter & 37 & 56 & 84 \\
\hline Grid spacing $(\Delta z=\Delta y)$ & 0.000268 & 0.000178 & 0.0001191 \\
\hline \multicolumn{4}{|l|}{ Newtonian } \\
\hline Velocity $[\mathrm{mm} / \mathrm{s}]$ & 2.296 & 2.290 & 2.286 \\
\hline Relative velocity difference (magnitude) [\%] & 0.437 & 0.175 & \\
\hline \multicolumn{4}{|l|}{ Odroyd-B $\left(D e_{T}=3.75, c=0.5\right)$} \\
\hline Velocity $[\mathrm{mm} / \mathrm{s}]$ & 2.146 & 2.113 & 2.085 \\
\hline Relative velocity difference (magnitude) [\%] & 2.926 & 1.343 & \\
\hline \multicolumn{4}{|l|}{$\operatorname{FENE-CR}\left(D e_{T}=30, c=0.5\right)$} \\
\hline Velocity $[\mathrm{mm} / \mathrm{s}]$ & 1.699 & 1.655 & 1.626 \\
\hline Relative velocity difference (magnitude) [\%] & 4.489 & 1.784 & \\
\hline
\end{tabular}

The effect of the time integration step has been investigated considering a Newtonian and viscoelastic case adopting mesh $M_{2}$. We executed different simulations by considering four different values of the maximum Courant number, namely, $C o_{\max }=0.1,0.04,0.02,0.01$ again for both a Newtonian-Newtonian system and a viscoelastic-Newtonian configuration using the Oldroyd-B constitutive equation to model the continuous phase (considering the same 
parameters adopted for the previous spatial refinement study). As pointed out previously, we observed a dependence of the droplet shape on the time step. In order to quantify the magnitude of the deformation relative to the circular shape, we define the droplet aspect ratio, $A R_{d}$, as the ratio between the droplet major and minor axes, $D_{1}$ and $D_{2}$ (unlike the case for the shear flow discussed before, where the droplet assumes ellipsoidal shapes, a Newtonian droplet migrating in a viscoelastic fluid can be affected by loss of fore-and-aft symmetry, therefore we found advisable to describe the droplet deformation adopting different quantities), respectively. Table III shows the values of $A R_{d}$ and the terminal droplet velocity for the four Courant numbers considered. We notice that the departure from the reference circular shape $\left(A R_{d}=1\right)$ decreases by decreasing the maximum time step allowable for the simulation. In particular, for $C o_{\max }=0.1$ the relative percentage deviation from the circular shape is $6.4 \%$, while reaches a minimum value of $0.8 \%$ for the smallest $C o_{\max }$. Additionally, we tested the effect of the grid spacing and noticed that by using a finer mesh, keeping the same maximum Courant number, does not have appreciable influence on the droplet shape. For completeness, we did the same tests also for the viscoelastic configuration. The results indicate a good convergence both in terms of deformation and the terminal velocity, when the time step is decreased (cf. Table II).

Table III: Effect of the time integration step on the droplet aspect ratio and terminal velocity. We consider a two-dimensional droplet for a Newtonian case and a viscoelastic case using the Oldroyd-B model. All the simulations have been carried out by employing mesh $M_{2}$. The relative differences have been evaluated considering the results obtained for $C o_{\max }=0.01$ as a reference.

$$
\begin{array}{llll}
\mathrm{Co}_{\text {Max }}=0.1 & \mathrm{Co}_{\text {Max }}=0.04 & \mathrm{Co}_{\text {Max }}=0.02 & \mathrm{Co}_{\text {Max }}=0.01
\end{array}
$$

\begin{tabular}{|c|c|c|c|c|}
\hline & $\mathrm{Co}_{\text {Max }}=0.1$ & $\mathrm{Co}_{\text {Max }}=0.04$ & $\mathrm{Co}_{\text {Max }}=0.02$ & $\mathrm{Co}_{\mathrm{Max}}=0.01$ \\
\hline \multicolumn{5}{|l|}{ Newtonian case } \\
\hline Droplet aspect ratio $D_{a}$ & 0.936 & 0.973 & 0.986 & 0.992 \\
\hline Relative aspect ratio difference [\%] & 5.60 & 1.90 & 0.60 & \\
\hline Terminal velocity $[\mathrm{mm} / \mathrm{s}]$ & 2.223 & 2.284 & 2.288 & 2.296 \\
\hline Relative velocity difference [\%] & 3.18 & 0.52 & 0.35 & \\
\hline \multicolumn{5}{|l|}{ Viscoelastic case } \\
\hline Droplet aspect ratio $D_{a}$ & 0.967 & 0.997 & $1.008^{*}$ & $1.014^{*}$ \\
\hline Relative aspect ratio difference [\%] & 7.70 & 4.00 & 0.60 & \\
\hline Terminal velocity $[\mathrm{mm} / \mathrm{s}]$ & 2.040 & 2.079 & 2.113 & 2.132 \\
\hline Relative velocity difference [\%] & 4.30 & 2.49 & 0.89 & \\
\hline
\end{tabular}


*The prolate shape is consistent with the presence of the viscoelastic stresses that act to deform the droplet along the direction of the motion.

In light of these results, we performed all subsequent three-dimensional simulations adopting a 3D equivalent of mesh $M_{2}$ and setting $C o_{\max }=0.02$, keeping in mind that the shapes we show might be affected by an uncertainty of the order of $1 \%$ or less. It is worth mentioning that the effect of the time step used to integrate Eq. (14) has also been investigated. In particular, we considered the configuration mesh $M_{2}$ and $C o_{\max }=0.02$ by changing systematically the number of sub-cycles (in a number of 2, 5 and 10). No appreciable differences in the shape were observed, providing evidence that the droplet shape was insensitive to the time step adopted for the integration of Eq. (14). 